\title{
Dynamics of a Mutual Inhibition Circuit between Pyramidal Neurons Compared to Human Perceptual Competition
}

\author{
Naoki Kogo, ${ }^{1}$ Felix B. Kern, ${ }^{2}$ Thomas Nowotny, ${ }^{3}$ Raymond van Ee, ${ }^{1,4}$ Richard van Wezel, ${ }^{1,5}$ and \\ Takeshi Aihara ${ }^{6}$ \\ ${ }^{1}$ Biophysics, Donders Institute for Brain, Cognition and Behaviour, Radboud University, Nijmegen, The Netherlands, 6525 AJ, ${ }^{2}$ School of Life \\ Sciences, University of Sussex, Brighton, United Kingdom, BN1 9QG, ${ }^{3}$ School of Engineering and Informatics, University of Sussex, Brighton, \\ United Kingdom, BN1 9QJ, ${ }^{4}$ Brain and Cognition, University of Leuven, Leuven, Belgium, 3000, ${ }^{5}$ Biomedical Signals and Systems, MedTech Centre, \\ University of Twente, Enschede, The Netherlands, 7522 NB, and ${ }^{6}$ Brain Science Institute, Tamagawa University, Tokyo, Japan, $194-8610$
}

Neural competition plays an essential role in active selection processes of noisy and ambiguous input signals, and it is assumed to underlie emergent properties of brain functioning, such as perceptual organization and decision-making. Despite ample theoretical research on neural competition, experimental tools to allow neurophysiological investigation of competing neurons have not been available. We developed a "hybrid" system where real-life neurons and a computer-simulated neural circuit interacted. It enabled us to construct a mutual inhibition circuit between two real-life pyramidal neurons. We then asked what dynamics this minimal unit of neural competition exhibits and compared them with the known behavioral-level dynamics of neural competition. We found that the pair of neurons shows bistability when activated simultaneously by current injections. The addition of modeled synaptic noise and changes in the activation strength showed that the dynamics of the circuit are strikingly similar to the known properties of bistable visual perception: The distribution of dominance durations showed a right-skewed shape, and the changes of the activation strengths caused changes in dominance, dominance durations, and reversal rates as stated in the well-known empirical laws of bistable perception known as Levelt's propositions.

Key words: bistable perception; double patch clamp; dynamic clamp; neural dynamics; perceptual organization; visual cortex

\section{Significance Statement}

Visual perception emerges as the result of neural systems actively organizing visual signals that involves selection processes of competing neurons. While the neural competition, realized by a "mutual inhibition" circuit has been examined in many theoretical studies, its properties have not been investigated in real neurons. We have developed a "hybrid" system where two real-life pyramidal neurons in a mouse brain slice interact through a computer-simulated mutual inhibition circuit. We found that simultaneous activation of the neurons leads to bistable activity. We investigated the effect of noise and the effect of changes in the activation strength on the dynamics. We observed that the pair of neurons exhibit dynamics strikingly similar to the known properties of bistable visual perception.

Received Sep. 23, 2020; revised Nov. 16, 2020; accepted Dec. 9, 2020.

Author contributions: N.K., F.B.K., T.N., R.v.E., R.v.W., and T.A. designed research; N.K. performed research; N.K., F.B.K., and T.N. contributed unpublished reagents/analytic tools; N.K. and F.B.K. analyzed data; N.K. wrote the first draft of the paper; N.K., F.B.K., T.N., R.v.E., R.v.W., and T.A. edited the paper; N.K. wrote the paper.

N.K. was supported by Fonds voor Wetenschappelijk Onderzoek FWO-Flanders Post Doc Grant 12L5115N (University of Leuven, 2014-2017) and Marie Curie Actions European Fellowship 794273 (Radboud University, 2018 to current). T.N. was supported by Engineering \& Physical Sciences Research Council EP/P006094/1 and European Union 785907. R.v.W. was supported by NWO-TTW Grants NESTOR and INTENSE. We thank Dr. Ginamaria Maccaferri (Northwestern University, Chicago) for supporting the project during the time of piloting; Dr. Andreas Burkhalter (Washington University, St. Louis) for providing detailed information of the anatomy of mouse visual cortex; Dr. Yoshikazu Isomura (Tamagawa University, Machida, Japan) for supporting to conduct the project and the anatomical analysis at Brain Science Institute; and Dr. Nael Nadif Kasri and Dr. Dirk Schubert (RadboudUMC, Nijmegen, The Netherlands) for supporting to run the experiments at their laboratory.

The authors declare no competing financial interests.

Correspondence should be addressed to Naoki Kogo at naoki.kogo@gmail.com.

https://doi.org/10.1523/JNEUROSCI.2503-20.2020

Copyright $\odot 2021$ the authors

\section{Introduction}

Visual perception is an emergent property resulting from an active organization of input signals by the brain while being subjected to the underrepresented, noisy, and ambiguous signals received by the eyes. In other words, the brain makes selections among neural signals representing the conflicting signals that are competing with each other. A well-known perceptual phenomenon representing signal competition and selection processes is "bistable perception," which occurs when visual signals support two likely perceptual interpretations. Signals that support one of the percepts are selected coherently at any given time and one percept becomes dominant. The input signals are eventually reorganized to establish the alternative percept, leading to reversals between the two percepts every few seconds (Leopold and 
Logothetis, 1999). This repetitive perceptual reorganization in bistable perception provides information about how visual signals are processed, organized, and eventually lead to conscious perception. The abundant literature on bistable perception is an important resource of information to investigate underlying neural mechanisms.

Neural competition is often modeled by "mutual inhibition" between neurons. A possible neural circuit is shown in Figure $1 a$, in which each pyramidal neuron (PN1 or PN2) activates a partner inhibitory neuron (IN1 and IN2, respectively), which, in turn, projects an inhibitory synapse to the competing PN, forming disynaptic inhibitory connections in both directions.

It has been suggested that the conflicting signals for local features, such as orientation (Sillito, 1975; Bonds, 1989), motion direction (Mikami et al., 1986; Snowden et al., 1991), and edge assignment (Zhou et al., 2000; Kogo and van Ee, 2015), compete with each other through such mutual inhibition circuits. This circuit has been implemented in computer models to explain bistable perception (Matsuoka, 1984; Mueller, 1990; Wilson, 1999; Wilson et al., 2000; Laing and Chow, 2002; Lankheet, 2006; Noest et al., 2007; Shpiro et al., 2009), object recognition (Masquelier et al., 2009), decision-making (Heuer, 1987; Usher and McClelland, 2001; Machens et al., 2005), and place cell field generation (Mark et al., 2017). It has also been suggested that these circuits underlie mechanisms, such as larger-scale neural interactions and feedback systems (Lee et al., 1999; Beck and Kastner, 2005; C. T. Wang et al., 2013) that establish a globally coherent percept. Moreover, disynaptic inhibitory connections between PNs are found in various layers and areas of neocortex (Kapfer et al., 2007; Ren et al., 2007; Silberberg and Markram, 2007; Berger et al., 2009) and hippocampus (Miles, 1990). It is hence possible that mutual inhibition serves as a canonical element of signal processing circuits in the brain. However, there have been alternative models for bistability and decision-making that implemented neural mechanisms different from mutual inhibition (Said and Heeger, 2013; Hayden and Moreno-Bote, 2018).

Despite the numerous theoretical models implementing mutual inhibition circuits, experimental tools are missing that allow thorough neurophysiological analysis of competing cortical neurons at the system-wide level because of the limitations of current technology. However, with the approach introduced in this paper, it is possible to construct a minimal unit of neural competition in real life. By investigating the neural dynamics of the minimal unit, considering it as a building block of the whole system, and comparing its dynamics with the ones of the whole system, it may be possible to deduce how neural elements are integrated into a whole system such that known behavioral properties emerge.

We established a technique where a model mutual inhibition circuit is implemented between a pair of two real-life PNs in brain slice preparations of mouse primary visual cortex (Fig. 1). The two neurons are patch-clamped and connected with each other via a computer model that allows them to interact in real time. This hybrid system has the advantage of keeping all physiological properties of the real PNs intact while providing full control over the computer-simulated connections between them.
Using this hybrid system, we succeeded to evoke bistable activity in the PNs. We investigated the dynamics of the bistable activity and compared them with the known dynamics of bistable visual perception, namely, the effects of noise and the effect of changing stimulus input intensity.

\section{Materials and Methods}

Experiments were performed at the Brain Science Institute (Tamagawa University) and the Donders Institute for Brain, Cognition and Behavior (Radboud University). The experimental animal procedures were approved by the Animal Research Ethics Committee of Tamagawa University (animal experiment protocol H29/08) and the Animal Ethics Committee of the Radboud University Nijmegen (DEC application 2018-0016). The procedures are in accordance with the Guidelines for Animal Experimentation in Neuroscience (Japan Neuroscience Society) and the Dutch legislation.

\section{Experimental design}

Brain slice preparation. Brain slices were prepared from the occipital part of the mouse brain that includes the visual cortex (strain C57BI6/J, age p12 to p24, both sexes). Mice were anesthetized deeply using isoflurane in an induction chamber. Following deep anesthesia, mice were quickly decapitated, and the brain was removed from the skull in a small container with chilled "cutting solution." For this process, the solution of either one of the following compositions was used (in $\mathrm{mM}$ ): $125 \mathrm{NaCl}, 25$ $\mathrm{NaHCO}_{3}, 2.5 \mathrm{KCl}, 1.25 \mathrm{NaH}_{2} \mathrm{PO}_{4}, 1 \mathrm{CaCl}_{2}, 2 \mathrm{MgCl}_{2}, 25 \mathrm{D}$-glucose, or 75 sucrose, $87 \mathrm{NaCl}, 25 \mathrm{NaHCO}_{3}, 2.5 \mathrm{KCl}, 1.25 \mathrm{NaH}_{2} \mathrm{PO}_{4}, 0.5 \mathrm{CaCl}_{2}$, $7 \mathrm{MgCl}_{2}, 25 \mathrm{D}$-glucose, both saturated with $95 \% \mathrm{O}_{2}, 5 \% \mathrm{CO}_{2}$. Then, the brain tissue was glued on to the cutting stage of a vibratome (VT1000S, Leica Microsystems, or Microm HM 650V, Thermo Fisher Scientific), submerged in the cutting solution above. Coronal or angled-coronal (Dong et al., 2004) sections of 300-400 $\mu \mathrm{m}$ thickness were cut and stored in an incubation chamber in $32^{\circ} \mathrm{C}-34^{\circ} \mathrm{C}$ for at least $30 \mathrm{~min}$, and then stored at room temperature until use.

Double whole-cell recordings. Slices were transferred to a recording chamber on a microscope stage and were superfused with ACSF maintained at a constant temperature $\left(32^{\circ} \mathrm{C}-34^{\circ} \mathrm{C}\right)$. ACSF had the following composition (in mM): $125 \mathrm{NaCl}, 25 \mathrm{NaHCO}_{3}, 2.5 \mathrm{KCl}, 1.25 \mathrm{NaH}_{2} \mathrm{PO}_{4}$, $2 \mathrm{CaCl}_{2}, 1 \mathrm{MgCl}_{2}, 25 \mathrm{D}$-glucose, saturated with $95 \% \mathrm{O}_{2}, 5 \% \mathrm{CO}_{2}$. The location of V1 was identified under the microscope (Olympus) equipped with differential interference contrast-infrared. Layers of visual cortex were identified; and the point where layer 5 starts thickening, going from medial to lateral, was used as a landmark of the border between V1 and LM (lateromedial area) (Q. Wang and Burkhalter, 2007), equivalent 
to V2 (Fig. 1c). All recordings were made from the region medial from the landmark. Under high magnification with $\times 40$ objective, PNs in layer $2 / 3$ were identified by their stereotypical morphology. In some cases, the recorded neurons were filled with biocytin; and postexperimental process indicated that, in all cases, they were PNs in layer $2 / 3$ (see below). Two neurons separated by at least $150 \mu \mathrm{m}$ distance were selected to reduce the probability of choosing connected pairs. Furthermore, experimental protocols were performed to check for monosynaptic (paired-pulse injection at $10 \mathrm{~Hz}$ to one of the neurons to evoke action potentials) and disynaptic connections (Kapfer et al., 2007; Silberberg and Markram, 2007) (100 Hz 11 pulses injection to one of the neurons to evoke a train of action potentials). None of the pairs reported in this paper was connected.

Pipettes for patch-clamp recordings were pulled from borosilicate thin glass capillaries (TW150-4, WPI) and filled with a filtered intracellular solution with the following composition (mM): $130 \mathrm{~K}$-gluconate, $10 \mathrm{KCl}, 4$ ATP-Mg, $0.3 \mathrm{Na}-\mathrm{GTP}, 10$ HEPES, and 10 phosphocreatine. For phosphocreatine, either $10 \mathrm{~mm} \mathrm{Na}_{2}$-phosphocreatine or a mixture of $5 \mathrm{mM} \mathrm{Na}_{2}$-phosphocreatine and $5 \mathrm{mM}$ Tris-phosphocreatine was used. The osmolarity of the solution was adjusted to 290-300 Osm by either Osmotron-5 (Orion Riken) or Semi-Micro Osmometer K-7400 (Knauer), and the $\mathrm{pH}$ was adjusted to 7.2. The final resistance of the pipettes was 7-9 $\mathrm{m} \Omega$. In some cases, biocytin was added to the pipette solution $(2.5-5 \mathrm{mg} / \mathrm{ml})$ to visualize the recorded neurons postexperimentally. Recordings were conducted using either two Axopatch 200B amplifiers or a Multiclamp 700 amplifier (both Molecular Devices). Data were lowpass filtered at $10 \mathrm{kHz}$ and were digitized at $20 \mathrm{kHz}$ using a Digidata A/D board model 1440A. Data were captured using the Clampex program suite (Molecular Devices). Series resistances were constantly monitored by injecting a $-100 \mathrm{pA}$ pulse in current-clamp configuration. Series resistances were balanced via a bridge circuit.

Cell identification. To visualize the PN pairs that were recorded, they were filled with biocytin by diffusion $(N=9)$. After the recording $(\sim 30$ $60 \mathrm{~min})$, the slices were kept in $4 \%$ PFA in PB solution, PBS $(0.1 \mathrm{M}, \mathrm{pH}$ 7.2), and were kept at $4^{\circ} \mathrm{C}$. After washing the tissue with PBS, it was quenched with $1 \% \mathrm{H}_{2} \mathrm{O}_{2}$ in $10 \%$ methanol and $90 \% \mathrm{PBS}$ for $5 \mathrm{~min}$. The tissue was washed with PBS and permeabilized with $2 \%$ Triton X-100 in PBS for $1 \mathrm{~h}$ and then put in $\mathrm{ABC}$ solution (ABC Elite Kit, Vector) overnight at $4^{\circ} \mathrm{C}$. After washing the tissue with PBS and then with Tris buffer $(0.05 \mathrm{M})$, it was processed with DAB solution $(0.5 \mathrm{~g} / 1$ in $0.05 \mathrm{M}$ Tris buffer), and $1 \% \mathrm{H}_{2} \mathrm{O}_{2}$ was added to enhance the reaction. After verifying the visualization of neurons, the tissue was washed by PBS and then mounted to glass slides with a mounting medium (Aquamount, Vector).

Dynamic clamp. A modified version of the dynamic clamp system StdpC (spike timing-dependent plasticity clamp) (Nowotny et al., 2006; Kemenes et al., 2011) was used to establish the connections between recorded neurons and model neurons with model synapses. The communication between the amplifier and StdpC was mediated by a National Instruments A/D board, model PCIe-6321. Dynamic clamp is a method whereby a modeled conductance (e.g., a synaptic or ionic conductance) is computed based on the measured membrane potential of a neuron, then injected into that neuron in real time with a patch-clamp electrode. Unlike other dynamic clamp systems which operate at fixed frequencies, StdpC does not require a real-time operating system, relying instead on precise measurement of the time elapsed in each measurecompute-inject cycle to perform the numerical integration of its models.

In addition to numerous improvements to the software interface, the following major additions were made to the previous version of StdpC (Nowotny et al., 2006). A passive membrane model was added, which can be augmented with models of ionic and synaptic conductances to form completely synthetic neuron models. To stabilize numerical integration of such models at StdpC's unpredictable and varying sampling frequency, the clamp cycle was upgraded from explicit Euler to a RungeKutta integration scheme of order 4/5. A number of performance enhancements were made to ensure high-frequency, and thus high-fidelity, updates to the injected current. A delay mechanism was added to the synapse models, allowing the simulation of conduction and synaptic delays. Finally, a model of synaptic background noise was added, reproducing the synaptic bombardment we would expect to see in vivo with
Table 1. Parameter sets for modeled ionic channels

\begin{tabular}{|c|c|c|c|c|c|c|c|c|}
\hline & $g_{\max }(\mathrm{nS})$ & $\begin{array}{l}V_{\text {rev }} \\
(\mathrm{mV})\end{array}$ & $\begin{array}{l}\text { Activation/ } \\
\text { Inactivation }\end{array}$ & Exponential & $\alpha / \beta$ & $F k(1 / \mathrm{ms})$ & $V(\mathrm{mV})$ & $s(\mathrm{mV})$ \\
\hline \multirow[t]{4}{*}{$\mathrm{Na}$} & 25,385 & 50 & Activation & 3 & $\alpha$ & 11.0 & -40 & -10 \\
\hline & & & & & $\beta$ & 24.0 & -65 & -18 \\
\hline & & & Inactivation & 1 & $\alpha$ & 20.07 & -65 & -20 \\
\hline & & & & & $\beta$ & 31.0 & -35 & -10 \\
\hline \multirow[t]{2}{*}{$K_{\mathrm{d}}$} & 615 & -100 & Activation & 4 & $\alpha$ & 10.1 & -55 & -10 \\
\hline & & & & & $\beta$ & 20.125 & -65 & -80 \\
\hline \multirow[t]{2}{*}{$K_{\mathrm{v} 3}$} & 3,000 & -100 & Activation & 1 & $\alpha$ & $1-0.12166$ & 4.18371 & -6.42606 \\
\hline & & & & & $\beta$ & 20.015857 & 0 & -25.4834 \\
\hline
\end{tabular}

statistically equivalent, randomly generated inhibitory and excitatory currents, as described in Effect of noise. The upgraded version of StdpC (version 6.1) is available at www.github.com/CompEphys-team/stdpc.

A custom-made summing circuit was used to combine the command signal from StdpC and the one from Clampex software, and the combined command signal was fed to the amplifier.

Hodgkin-Huxley models of ionic channels (conventional sodium, delayed rectifier potassium, and Kv3 potassium channels) were given to the model IN (mIN; membrane capacitance $0.2115 \mathrm{nF}$, leak conductance $63.462 \mathrm{nS}$, equilibrium potential for the leak conductance $-70 \mathrm{mV}$ ) (Pospischil et al., 2008). A Kv3 channel was added to simulate fast spiking INs (Lien and Jonas, 2003). The models are based on an " $\alpha / \beta$ formalism" as follows (see www.github.com/CompEphys-team/stdpc/tree/ master/manual):

$$
\begin{gathered}
I=g_{\text {max }} m^{p} h^{q}\left(V-V_{\text {rev }}\right) \\
\frac{d m}{d t}=\alpha_{m}(1-m)-\beta_{m} m \\
\alpha_{m}=k_{\alpha, m} F_{\alpha, m}\left(\frac{V-V_{\alpha, \mathrm{m}}}{s_{\alpha, m}}\right) \\
\beta_{m}=k_{\beta, m} F_{\beta, m}\left(\frac{V-V_{\beta, m}}{s_{\beta, m}}\right)
\end{gathered}
$$

(and analogous for $h$ ).

Here, $m$ and $h$ are activation and inactivation variables. $g_{\max }$ is the maximum conductance of the ion channel, and $V_{\text {rev }}$ is the reversal potential of the ion. The form of the function $F$ is either one of the three below:

$$
\begin{gathered}
F_{1}(x)=\frac{x}{\exp (x)-1} \\
F_{2}(x)=\exp (x) \\
F_{3}(x)=\frac{1}{1+\exp (x)}
\end{gathered}
$$

For the potassium channels, the formalisms are the same, except that no inactivation components are included. The form of the function $F$ and the parameters for $\alpha$ and $\beta$ for the individual components are as summarized in Table 1 . These parameter values were taken from Pospischil et al. (2008) for basic membrane properties, from Hodgkin and Huxley (1952) for sodium and delayed rectifier potassium channels, and from Lien and Jonas (2003) for the Kv3 channel.

Conductance of excitatory and inhibitory synaptic events was modeled using the ChemSyn model in StdpC, following the equations and parameters described as follows:

$$
I=g_{s y n} S(t)\left(V_{\text {syn }}-V_{\text {post }}(t)\right)
$$




$$
\begin{gathered}
\tau_{\text {syn }} \frac{d S(t)}{d t}=\frac{S_{\infty}\left(V_{\text {pre }}(t)\right)-S(t)}{1-S_{\infty}\left(V_{\text {pre }}(t)\right)} \\
S_{\infty}\left(V_{\text {pre }}(t)\right)=\left\{\begin{array}{cc}
\tanh \left(\frac{V_{\text {pre }}(t)-V_{T H}}{V_{\text {slope }}}\right) & \text { if } V_{\text {pre }}(t)>V_{T H} \\
0 & \text { otherwise }
\end{array}\right.
\end{gathered}
$$

Table 2. Parameter sets for synaptic conductance

\begin{tabular}{lllllll}
\hline & $g_{\text {syn }}(\mathrm{nS})$ & $V_{\text {syn }}(\mathrm{mV})$ & $\tau_{\text {syn }}(\mathrm{ms})$ & $V_{\text {TH }}(\mathrm{mV})$ & $V_{\text {slope }}(\mathrm{mV})$ & Synaptic delay $(\mathrm{ms})$ \\
\hline Excitatory & 10 & 0 & 5 & -20 & 25 & 1.0 \\
Inhibitory & 10 & -70 & 100 & -20 & 25 & 1.0 \\
\hline
\end{tabular}

Parameters for excitatory and inhibitory synapses are shown in Table 2. $g_{\text {syn }}$ for EPSP was selected so that it evokes an action potential in mINs (Fig. 2), and $g_{s y n}$ and $\tau_{s y n}$ for IPSP were selected to ensure strong enough suppression of target PN. The synaptic delay was set to $1 \mathrm{~ms}$ in all cases, and no synaptic plasticity was included in the model.

Disynaptic mutual inhibition connections. Establishment of a mutual inhibition circuit was verified as follows. Injection of a brief (1 ms) depolarization current (1500-2000 pA) to one of the pairs of PNs evoked an action potential (Fig. $2 a$, red and blue triangles), which triggered an excitatory synaptic conductance in the mIN. This synaptic event evoked an EPSP in the IN. As shown in Figure $2 a$, when $g_{\text {syn }}$ was set to $10 \mathrm{nS}$ or higher, the EPSP evoked an action potential (red and blue disks). This action potential in the IN triggered an inhibitory synaptic conductance, which was fed to the postsynaptic $\mathrm{PN}$ as an injected IPSC via the amplifier, giving rise to a corresponding IPSP (blue and red asterisks). Figure $2 a$ shows that an action potential was first evoked in the $\mathrm{PN} 1$ (PN1) and the PN 2 (PN2) received an IPSP. Later, an action potential was evoked in PN2 that resulted in an IPSP given to PN1, illustrating that the mutual inhibition circuit was established between the two PNs by this system. As shown in Figure $2 b$, the INs show trains of action potentials corresponding to the action potentials of presynaptic PNs during bistable activity.

Bistable activity. Bistable activity in a pair of PNs is evoked by the following protocol. First, before the dynamic clamp-mediated model circuit is switched on, depolarization currents that evoke action potentials at $\sim 10 \mathrm{~Hz}$ in the two neurons are determined separately. Next, the model circuit is switched on to activate the mutual inhibitory connection, and the depolarization currents as determined above are injected. In most cases, this already produces bistable activity in the pair (unless one of the neurons is $100 \%$ dominant). However, every neuron has different firing patterns, different degrees of responses to given synaptic inputs, and different sizes of action potentials (which influence the strength of postsynaptic events). As a result, the bistable activity often does not show equal dominance between the two neurons, although the firing rates are equivalent between them. Therefore, in the case that it is necessary to find the current pair where the dominance of the two neurons is approximately equal ( $50 \%$ dominance point), the currents are further adjusted by either increasing the current in the weaker neuron or decreasing the current in the stronger neuron.

Dominance, dominance durations, and reversal rates were calculated using custom MATLAB (The MathWorks) scripts. Unlike behavioral studies, in which a dominant percept is indicated as a continuous signal (by button press), the dominance of a neuron is signaled by sustained repetitive firing of action potentials. Hence, we defined the "dominance duration" of a neuron as follows (illustrated in Fig. 3). First, a continuous firing of action potentials in one neuron until an action potential occurs in the other neuron is considered as a tentative dominance duration of the neuron (Fig. $3 b$ ). Hence, at this stage, the dominance durations of the two neurons are mutually exclusive. There are short dominance durations (blue asterisks for PN2 and red asterisk for PN1). There are also a series of alternations of short dominance durations between the two neurons (green asterisks). Next, dominance durations shorter than $250 \mathrm{~ms}$ are eliminated (Fig. 3c). This process results in short lags between the dominance durations (blue and red asterisks). The occurrence of the short lag is not considered as reversal; hence, the previous dominance is considered to continue (arrows). These processes result in the final dominance durations without short durations (Fig. $3 d$ ). There are also the intervals that are not assigned to either of the neurons corresponding to the period marked with green asterisks in Figure $3 c$. This is because alternating short durations occur between the two neurons during these periods (Fig. $3 c$, green asterisks). These periods are assigned as "both active" (Fig. $3 d$, bottom). Dominance and reversal rates were computed based on this definition of dominance durations. "Dominance" of a neuron is defined as the ratio of total dominance durations of the neuron (sum of all dominance durations of the neuron) divided by the sum of the total dominance durations of both neurons. A reversal is defined as the dominance switching from one neuron to the other, regardless of the presence or absence of a "both active" phase during the switch. The coefficient of variation of dominance durations was computed according to Pastukhov and Braun (2011).

Special attention was paid to the recording conditions. If the following criteria were not met, the recording was halted: The overshoot of 


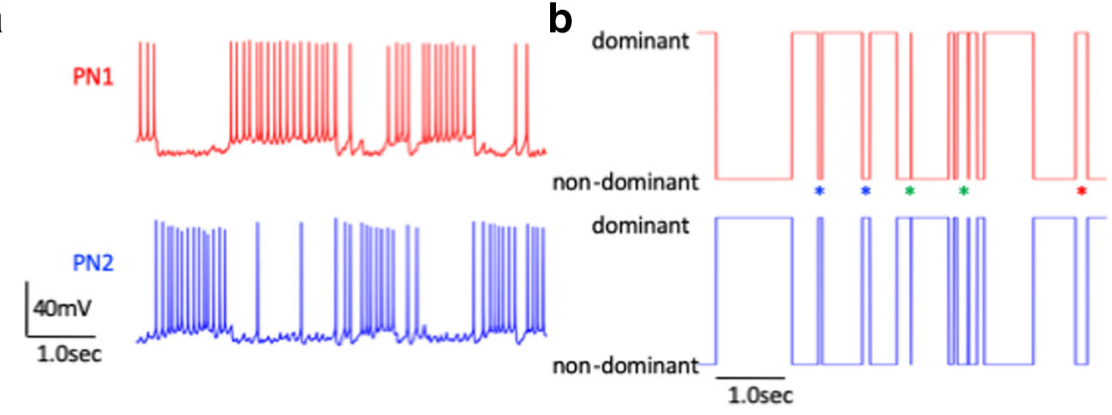

C

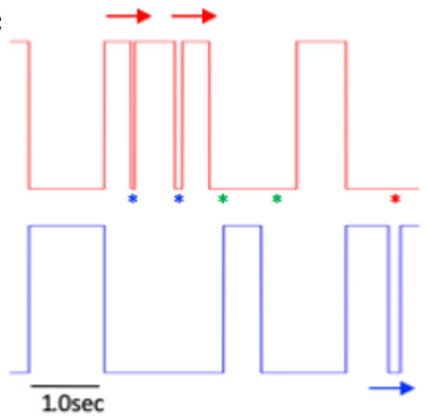

d

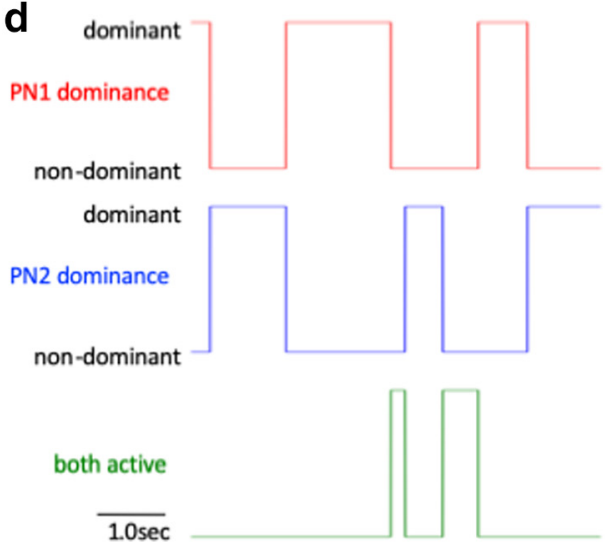

Figure 3. Computation of dominant durations. $\boldsymbol{a}$, A part of a recording of bistable activity. $\boldsymbol{b}$, First step computation of dominance durations. Here, continuous firing of action potentials in one neuron until an action potential occurs in the other neuron is considered as a tentative dominance duration of the first neuron. Hence, the dominant durations of the two neurons are mutually exclusive. There are short dominant durations (blue asterisks for PN2 and red asterisk for PN1). There are also series of alternations of short dominant durations between the two neurons (green asterisks). $c$, Dominance durations after choosing only long durations (longer than $250 \mathrm{~ms}$ ). This process results in short lags between the dominance durations (blue and red asterisks). There are also the intervals that are not assigned to either of the neurons corresponding to the period marked with green asterisks in $\boldsymbol{b}$. The short lags are not considered as reversals; hence, the previous dominance is considered to continue (arrows). $\boldsymbol{d}$, These processes result in the final dominance durations without short durations. And the periods not assigned to neither of the neurons are assigned as "both active" (bottom).
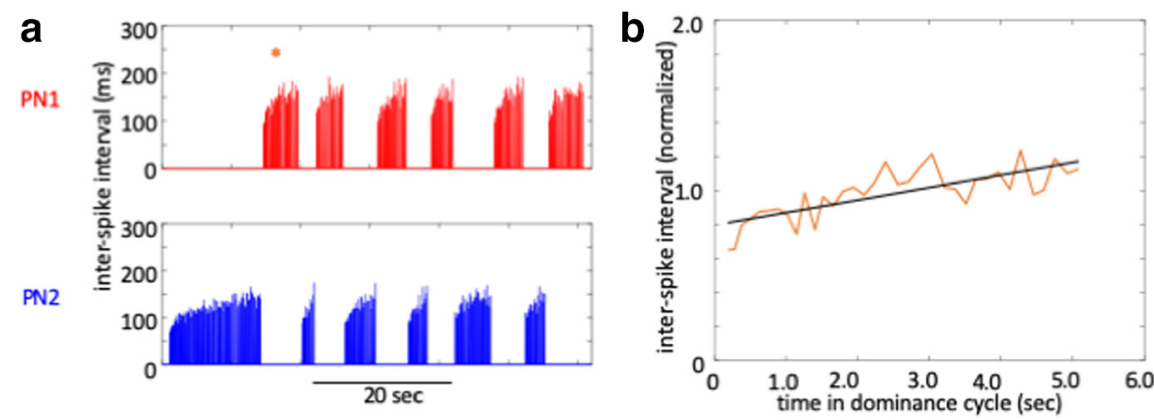

Figure 4. $\quad \boldsymbol{a}$, Interspike intervals of an example shown in Figure $5 a$. $\boldsymbol{b}$, Linear regression (black) of interspike intervals (orange plot) taken from the first cycle of PN1 (orange asterisk in $\boldsymbol{a}$ ) plotted over time from the onset of its dominance duration.

action potential should be higher than $10 \mathrm{mV}$, and changes in the size of the action potential, in series resistance, and in firing rate to a given depolarization current should be $<15 \%$ during data collection.

Analysis of adaptation. Interspike intervals and the peaks of action potentials were estimated with custom MATLAB scripts. Upon detection of action potentials, interspike intervals and the peaks were measured. These values were plotted against time to visualize the progress of adaptation within individual dominance episodes. To pool the data, first, the time from the onset of the dominance cycle to the end of this cycle was normalized by dividing it by the cycle's dominance duration (for the individual cycles of the individual pairs), resulting in the normalized time ranging from 0 to 1 . Second, interspike intervals and the magnitude of action potential peaks were normalized by the first values of the individual cycles. Third, the normalized values across all pairs were sorted into bins of size 0.01 . Finally, the mean and SD of all interspike intervals and action potential peaks in a given bin were plotted against the normalized time. As an indicator of the progress of adaptation, interspike intervals (normalized by the mean of individual pair) were plotted over time from the onset of each dominance cycle (Fig. 4a), and linear regression was applied to the plot (Fig. 4b). This resulted in slope values that indicated the change of interspike intervals. To pool the data, the dominance durations of individual pairs were normalized by their mean values and the slopes, normalized by the mean values of individual neurons, were plotted over the normalized duration.

Effect of noise. To investigate the effect of noise on the dynamics of bistable activity, synaptic background activity was simulated according to the model by Destexhe et al. (2001). In their simulation, random walklike fluctuations of membrane conductance were modeled by applying the OrnsteinUhlenbeck model of Brownian motion (Uhlenbeck and Ornstein, 1930). Their formalism of synaptic noise was implemented in the StdpC dynamic clamp system. The evolution of the simulated synaptic noise depends on the noise time constant $\tau$, which controls noise color, as well as the mean $g_{\text {mean }}$ and SD $\left(S D_{g}\right)$ of the noise, and is modeled as follows:

$$
I=g(t)\left(V_{r e v}-V\right)
$$

$$
\begin{gathered}
g(t+\Delta t)=g_{\text {mean }}+\left(g(t)-g_{\text {mean }}\right) e^{-\frac{\Delta t}{\tau}}+A r \\
A=\sqrt{\frac{D \tau\left(1-e^{\left.-\frac{2 \Delta t}{\tau}\right)}\right.}{1-e^{2}}}
\end{gathered}
$$

Here, $r$ is a pseudo-random number drawn from a normal distribution with mean 0 and SD 1 , and the noise diffusion coefficient $D$ is related to the noise SD as follows:

$$
\frac{D=2 S D g^{2}}{\tau}
$$

Excitatory and inhibitory synaptic noise is modeled separately. The level of noise is expressed as the $S D_{g}$ of the synaptic conductance and systematically manipulated, whereas the average conductance $g_{\text {mean }}$, which functions as a constant current offset, remained unchanged. The amount of noise given to mINs was larger than that given to PNs because PNs already have intrinsic synaptic noise (see Fig. $7 a$ ) from their presynaptic neurons within the brain slice. The standard parameter set (used as default unless mentioned otherwise) for the noise is shown in Table 3. 
Table 3. Parameter sets for synaptic noise

\begin{tabular}{llclcc}
\hline & Excitatory/inhibitory & $g_{\text {mean }}(\mathrm{nS})$ & $S_{g}(\mathrm{nS})$ & $V_{\text {rev }}(\mathrm{mV})$ & $\tau(\mathrm{ms})$ \\
\hline PN & Excitatory & 0 & 0.2 & 0 & 5 \\
& Inhibitory & 0 & 0.4 & -70 & 10 \\
\multirow{2}{*}{$\mathrm{N}$} & Excitatory & 2 & 1.0 & 0 & 5 \\
& Inhibitory & 10 & 2.0 & -70 & 10 \\
\hline
\end{tabular}

In the experiments for the effect of noise level and the effect of activation level (below), the length of each trial was $200 \mathrm{~s}$ with 193.5-s-long depolarization current.

Effect of short-term plasticity. It has been reported that there are diverse and cell-type-specific short-term plasticity effects in neocortex and hippocampus (for review, see Markram et al., 2004; Silberberg et al., 2005; Tremblay et al., 2016; Pelkey et al., 2017). To explore its effect on bistable activity, short-term depression (STD) was implemented in the model synaptic conductance in StdpC by introducing a depression factor, $h$, defined as follows:

$$
\begin{gathered}
I=g_{\text {syn }} S(t) h(t)\left(V_{\text {syn }}-V_{\text {post }}(t)\right) \\
\tau_{h} \frac{d h(t)}{d t}=h_{\infty}\left(V_{\text {pre }}(t)\right)-h(t) \\
h_{\infty}\left(V_{\text {pre }}\right)=\frac{1}{1+\exp \left(\frac{V_{\text {pre }}-V_{\text {Thresh }, h}}{V_{\text {Slope }, h}}\right)} \\
\tau_{h}\left(V_{\text {pre }}\right)=\tau_{0}-\frac{\tau_{\text {Ampl }}}{1+\exp \left(\frac{V_{\text {pre }}-V_{\text {Thresh }, \tau}}{V_{\text {Slope }, \tau}}\right)}
\end{gathered}
$$

STD was modeled in both excitatory synapses from PNs to fast spiking neurons and to inhibitory synapses from fast spiking neurons to PNs with the following parameters.

$$
\begin{gathered}
V_{\text {Thresh }, h}=-20 \mathrm{mV}, \\
V_{\text {Slope }, h}=2 \mathrm{mV}, \\
\tau_{0}=2.5 \mathrm{~ms}, \\
\tau_{\text {Ampl }}=-0.8, \\
V_{\text {Thresh }, \tau}=-20 \mathrm{mV}, \\
V_{\text {Slope }, \tau}=2 \mathrm{mV}
\end{gathered}
$$

Depression of PSPs was verified by generating a train of artificial spikes within StdpC by means of a spike generator (SG), connected by model synapses to a model neuron where EPSPs were evoked (see Fig. $11 a)$. The effect of STD was observed with two synaptic strengths $\left(g_{\text {syn }}=\right.$ $20 \mathrm{nS}$ and $g_{s y n}=50 \mathrm{nS}$ ) applied to all synapses in the circuit, chosen to demonstrate both successful mutual inhibition $\left(g_{s y n}=50 \mathrm{nS}\right)$, and failed mutual inhibition $\left(g_{s y n}=20 \mathrm{nS}\right)$ because of insufficient activation of the INs.

Paradigms equivalent to Levelt's experiments. For our experiments associated with the classic behavioral experiments of Levelt (Levelt, 1965; for generalized Levelt's propositions covering the full-range of stimulus intensities, see also Brascamp et al., 2006, 2015; Moreno-Bote et al., 2010), we systematically varied the strength of the sustained depolarization current into one, or both, of the PNs. Concerning the generalized Levelt's proposition I-III, the two currents were set as follows. First, the currents were set to evoke $10 \mathrm{~Hz}$ firing rate in the individual neurons (without mutual connections). Next, the modeled mutual inhibition circuit was activated while maintaining the static current injections. Then, only one of the two currents (randomly selected) was altered. The change of the current was made by steps of 10 or $20 \mathrm{pA}$.

In the analyses, the current that would evoke $50 \%$ dominance $\left(I_{50 \%}\right)$ was estimated by linear regression of dominance over the changing current. The change of the current is reported with reference to this control current value, defined as follows:

$$
\Delta I_{50 \% \text { orm }}=\frac{I-I_{50 \%}}{I_{50 \%}}
$$

Hence, in the plots in Figure $8 c-h$, the right side from $x=0$ indicates that the neuron that received the changing current was more dominant (stronger) than the other neuron, and the left side indicates the former being weaker than the latter. Before pooling the data $(N=46)$ for average durations, average dominance durations of individual trials were computed and were divided by the maximum average duration within individual neuron. To pool the data for the reversal rate, data were normalized by the maximum reversal rate of the individual pair.

Concerning the generalized Levelt's proposition IV, both currents were modified. First, a current pair that evoked a $10 \mathrm{~Hz}$ firing rate in the two neurons was found. If necessary, the current was adjusted until the current pair evoked $\sim 50 \%$ dominance. This current pair was considered as a control and is called $I_{10 \mathrm{~Hz}}$ (it is called as such for convenience, although the current pair did not always evoke $10 \mathrm{~Hz}$ firing). Next, in one of the two neurons, the current was changed with 10 or $20 \mathrm{pA}$ steps, and the current for the other neuron was changed proportionally. To pool the data, the change of the current is reported with reference to $I_{10 \mathrm{~Hz}}$, defined as follows:

$$
\Delta I_{10 \mathrm{Hznorm}}=\frac{I-I_{10 \mathrm{~Hz}}}{I_{10 \mathrm{~Hz}}}
$$

To pool the data for the reversal rate $(N=32)$, data were normalized by the reversal rate of the individual pair when the control current pair was used.

To make the bar plots of the pooled data (see Fig. $8 f-h$, right; Fig. $10 c$, bottom), the $\Delta I$ was binned, and the values in the individual bins were averaged. The order of trials with different current pairs was pseudo-randomly chosen.

\section{Statistical analysis}

For statistical analysis, repeated-measures ANOVA was applied using SPSS (IBM). Pairs with the standard noise parameter set for the experiment of noise $(N=15)$, pairs with injected current of $I_{50 \%}$ in Levelt I to III paradigms $(N=46)$, and pairs with injected current of $I_{10 H z}$ in Levelt IV paradigm $(N=32)$ are collectively called a "control pair," and statistical analyses were performed on these 93 pairs to report basic properties of bistability and adaptation. Error bars in the plots are \pm SEM.

All data and MATLAB codes for data analyses are published at (https://www.dropbox.com/sh/zytf4uzoxtvvqdq/AACulkZECAa4fgfyKtl fYIpwa?dl=0).

\section{Results}

Double patch-clamp recordings were performed from visually identified PNs in layer $2 / 3$ of mouse primary visual cortex (Fig. 1c). In total, 93 pairs of PNs from 32 mice were recorded. By using biocytin-filled patch pipettes, some PN pairs were labeled and visualized after the experiments $(N=9)$. In all cases, the stereotypical morphology of PNs (with a short apical dendrite and thin multiple oblique dendrites) was identified, located in layer $2 / 3$ of V1 (Fig. 1d).

\section{Bistable activity}

Mutual inhibitory connections between each pair of PNs were constructed by the StdpC dynamic clamp system (for details, see 
a

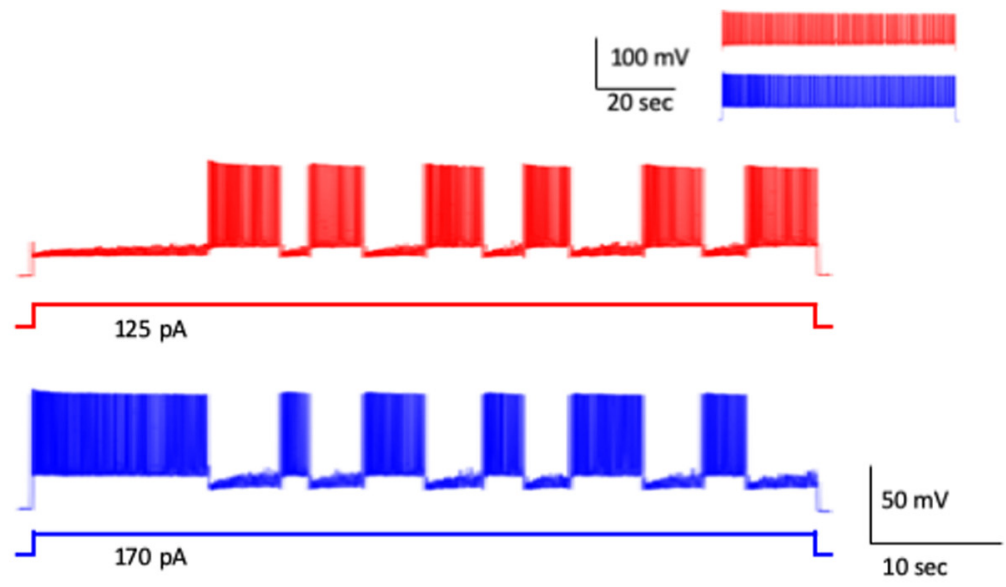

b
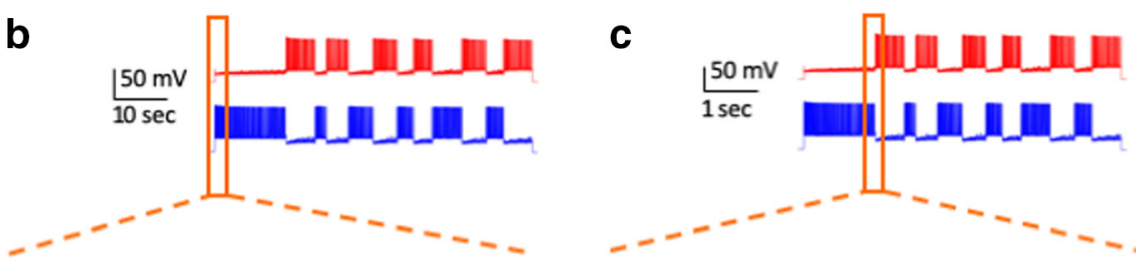

PN1
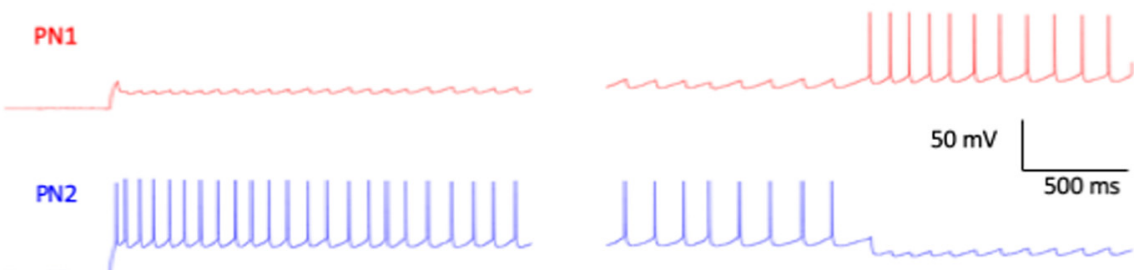

Figure 5. Evoked bistable activity in a pair of PNs with mutual inhibition connections. $\boldsymbol{a}$, Continuous injection of depolarization currents into the two PNs produces bistable activity with alternating dominance between them. MP, Membrane potential $(\mathrm{mV})$; $M C$, membrane current $(\mathrm{pA})$. Inset, The response of the same PNs to the same depolarization current injection without the mutual inhibition circuit, showing sustained continuous firing of action potentials. $\boldsymbol{b}$, The part of data (orange rectangle) shown in $\boldsymbol{a}$. Upon the onset of the current injection, both neurons started to depolarize but fired an action potential first. As a result, PN1 received an IPSP, causing PN2 to become dominant and PN1 suppressed. c, The part of data (orange triangle) around the time of reversal. The interspike interval increased during the dominant period of PN2 because of adaptation. Just after the rightmost action potential of PN2, PN1 got a sufficient time to recover from its IPSP, enabling it to reach its firing threshold before PN2 was able to fire its next action potential. The action potential of PN1 now resulted in an IPSP in PN2 entailing a reversal of dominance.

Materials and Methods). When continuous depolarization currents were injected into PN1 and PN2 simultaneously, bistable activity with alternating dominance between the two PNs was evoked as shown in Figure $5 a$. Figure $5 b$ shows the details of the onset of the response to the current injection on a shorter time scale. Both neurons started to depolarize at the onset, but PN2 reached the action potential threshold before PN1; hence, PN1 received the evoked IPSP before succeeding to generate an action potential. Thereafter, PN2 showed sustained firing of action potentials, and it achieved initial dominance. An increase of interspike intervals in the dominant neuron is visible. Also, there is a ramp-like slow depolarization of the suppressed neuron (Fig. $5 a$ ). The former is a sign of neural adaptation, whereas the latter indicates both the recovery of the neuron from adaptation as well as the recovery of the membrane potential from IPSPs because of the gradual increase of the interspike intervals. Figure $5 c$ shows data from when the reversal of dominance occurred. With the continuous increase of interspike interval in PN2, PN1 recovered more and more from the received barrage of IPSPs. The interspike interval of PN2 eventually became long enough such that the membrane potential of $\mathrm{PN} 1$ reached the action potential threshold before PN2 could generate an action potential. Consequently, PN2 received an IPSP evoked by the first action potential of PN1. From then on, PN1 became dominant and PN2 became suppressed.

\section{Adaptation and dominance durations}

To investigate the role of adaptation in the mutual inhibition competition process, we analyzed neurophysiological properties that reflect adaptation: interspike intervals and peaks of action potentials are plotted in Figure $6 a$ and Figure $6 b$, respectively, for the example bistable activity shown in Figure $5 a$. Normalized values are pooled for the "control pairs" ( $N=93$, for the definition of the "control pairs," see Materials and Methods) and plotted over normalized dominance durations in Figure $6 c$ and Figure $6 d$, for interspike intervals and action potential peaks, respectively. The results indicate monotonic changes (increase of interspike intervals and decrease of action potential peaks over time) while a neuron is dominant. Furthermore, there are clear correlations between the dominance durations and the changes of the interspike intervals. We applied a linear regression to interspike intervals as a function of time in the dominance duration (see Fig. 4). The slope indicates how quickly the adaptation progresses. As shown in Figure $6 e$ (for the example shown in Fig. 5a) and Figure $6 f$ (for the pooled data of the control pairs), the slopes and the dominance durations were inversely correlated (repeated-measures ANOVA for the pooled data $F_{(3,15)}=19.518$, $p<0.0001)$. Hence, when adaptation progresses quickly, the dominance duration is bound to be shorter, indicating a role for adaptation in dominance reversals.

\section{Effect of noise}

Because of the stochasticity of dominance durations (Brascamp et al., 2006; Kim et al., 2006; Moreno-Bote et al., 2007; Huguet et al., 2014; Pisarchik et al., 2014), it has been argued that noise plays an important role for the reversal in bistable perception. To investigate the role of noise on the dynamics of bistability, we implemented an algorithm in the dynamic clamp system to introduce simulated noise of the synaptic conductance (Destexhe et al., 2001; Delgado et al., 2010). The noise was given to both PNs and $\mathrm{mINs}$ in the form of random fluctuations of excitatory and inhibitory synaptic conductance (for details, see Materials and Methods). Figure $7 a$ shows the baseline membrane potential of a $\mathrm{PN}$, and Figure $7 b$ shows the result of adding the modeled synaptic noise to it (all at $-60 \mathrm{mV}$ ). Next, the level of noise was changed systematically while the two PNs were exhibiting bistable activity as shown in Figure $7 c$ (the parameter sets for different noise level are shown in Fig. $7 d$, table). The results indicate that increased noise caused an increase of the reversal rate $\left(F_{(19,171)}=50.868, p<0.0001\right)$. The pooled data from 15 pairs of PNs are shown in Figure $7 d$. 
It is known that, in brain slice preparations, the amount of synaptic noise in individual neurons is much less than what is observed in intact brain preparations because of the cutoff of axons and lesser spontaneous activity in slice preparations (Destexhe et al., 2001). Therefore, to reproduce the intact brain environment, we use a parameter set of modeled excitatory and inhibitory synaptic noise, which will be called the "standard noise parameter set” (Fig. $7 c$, asterisk; Fig. $7 d$, table) from here on. For the rest of the experiments, the standard noise parameter set was used. The histogram of dominance durations of a $600 \mathrm{~s}$ recording of bistable activity with the standard parameter set is shown in Figure 7e. It shows a skewed distribution as stereotypically observed in bistable perception. The average of dominance durations and reversal rates of the 15 pairs with the standard noise parameter set were $7.7 \pm 5.6 \mathrm{~s}$ and $12.0 \pm 10.5 \mathrm{~min}^{-1}$, respectively. These values for the control pairs $(N=93)$ were $8.2 \pm 7.8 \mathrm{~s}$ and $11.5 \pm$ $10.8 \mathrm{~min}^{-1}$, respectively. The coefficient of variation of dominance durations of the control pairs was $0.60 \pm 0.21$.

\section{Effect of current intensity ("generalized Levelt paradigms")}

A set of widely replicated empirical laws from the perceptual competition literature, known as Levelt's propositions, describes the relationship between the strengths of two competing stimuli and the dynamics of their bistable perception (Levelt, 1965) in terms of dominance, dominance duration, and reversal rate. Furthermore, Brascamp et al. (2015) and Moreno-Bote et al. (2010) reported a generally accepted updated version of Levelt's propositions so that the description of bistable dynamics covers the full range of stimulus strengths (Levelt's original propositions were based on the range of stimulation where the stimulus strength of one of the two input signals increased; and hence, the effect of decreasing the strength was not included). To compare the dynamics of the pairs of mutually inhibited PNs to the generalized Levelt's propositions, we injected sustained depolarization currents and systematically varied (increased and decreased) the strength of the current into one, or both, of the PNs (Fig. 8a).

To examine the first three generalized propositions of Levelt, the current injected into one of the two neurons was varied while the current injected into the other neuron was kept constant (Fig. $8 b$ ). In total, 46 pairs were recorded with this paradigm. To pool the data, first, the current that would evoke $50 \%$ dominance (the total period that one neuron is dominant over the other is equal for both neurons) was estimated $\left(I_{50 \%}\right)$ by linear regression of dominance over the changed current. The change of the current is reported with reference to this control current value (i.e., 0 in abscissa indicates the current pair that would evoke $50 \%$ dominance). Hence, in the plots shown in Figure $8 c-h$, the indicate \pm SEM. b

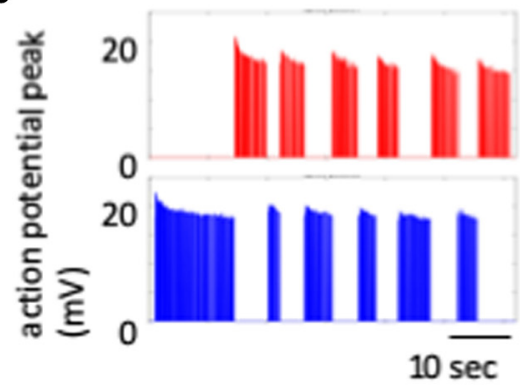

d

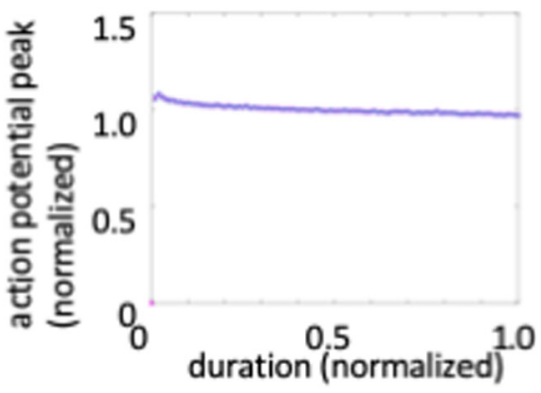

f

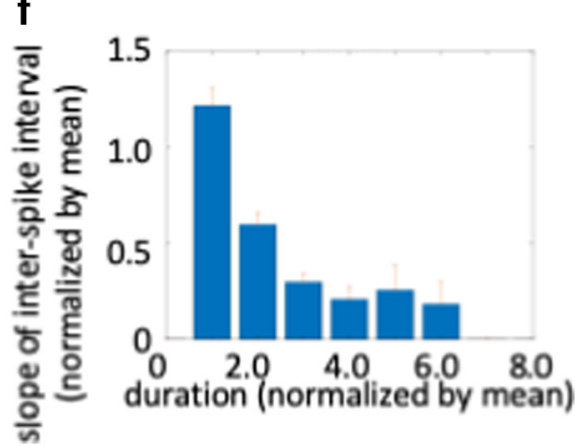

Figure 6. Adaptation of dominant neuron and its correlation to dominance duration. $\boldsymbol{a}, \boldsymbol{b}$, The physiological signatures of dominance episodes. $\boldsymbol{c}, \boldsymbol{d}$, Average of interspike intervals (c) and the action potential peaks (d) for pooled data of all 93 "control pairs" (for the definition, see Materials and Methods). $\boldsymbol{e}$, Slope of interspike interval as a function of dominance duradominance duration in the pooled data. The dominance durations of individual pairs were normalized by their mean values before pooling. The normalized duration was binned and the pooled data were averaged for the individual bins. Error bars

neurons with the changing injected current are more dominant ("stronger") on the right side of the plot from 0 , whereas on the left side, they are less dominant ("weaker").

We first tested the generalized Levelt's proposition I: Increasing stimulus strength for one of the competing stimuli will increase the perceptual dominance of that stimulus. Figure $8 c$ depicts the change of the dominance ratios of the two PNs over injected current (with reference to $I_{50 \%}$ of PN1) for the example shown in Figure $8 b$. There is a clear trend of increase of dominance of PN1 whose current was increased (red) and of decrease of dominance of PN2 whose current was kept constant (blue). Figure $8 f$ shows pooled data $(N=46)$ for the dominance ratio, replicating that there is an increasing dominance of the neurons whose currents were increased (red, $F_{(6,24)}=15.558, p<0.0001$ ), and decreasing dominance for their counterparts whose currents were kept constant (blue, $F_{(6,24)}=15.558, p<0.0001$ ). This is in line with the generalized Levelt's proposition I.

Levelt's proposition II states: Increasing the difference in stimulus strength between the two competing stimuli will 
a

PN1

b

PN2

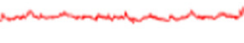

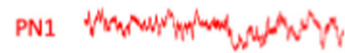
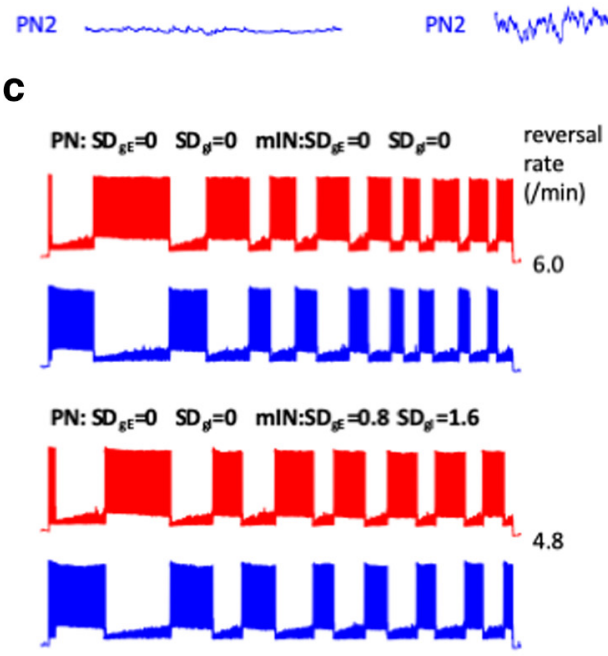

- $\mathrm{PN}: \mathrm{SD}_{\mathrm{gE}}=0.2 \mathrm{SD}_{\mathrm{g}}=0.4 \mathrm{mIN}: \mathrm{SD}_{\mathrm{gE}}=1.0 \mathrm{SD}_{\mathrm{g} i}=2.0$
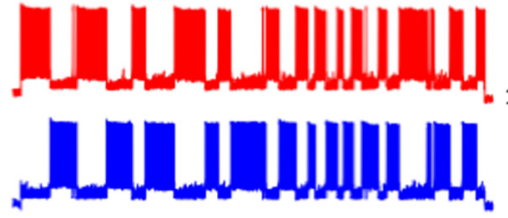

$\mathrm{PN}: \mathrm{SD}_{\mathrm{gE}}=0.4 \mathrm{SD}_{\mathrm{gg}}=0.8 \mathrm{mIN}: \mathrm{SD}_{\mathrm{gf}}=1.2 \mathrm{SD}_{\mathrm{gl}}=2.4$
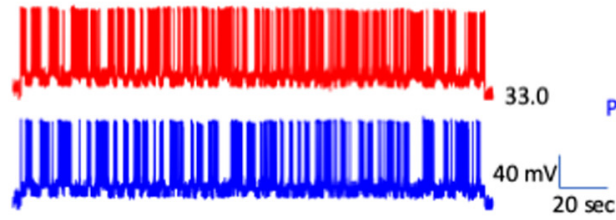

d
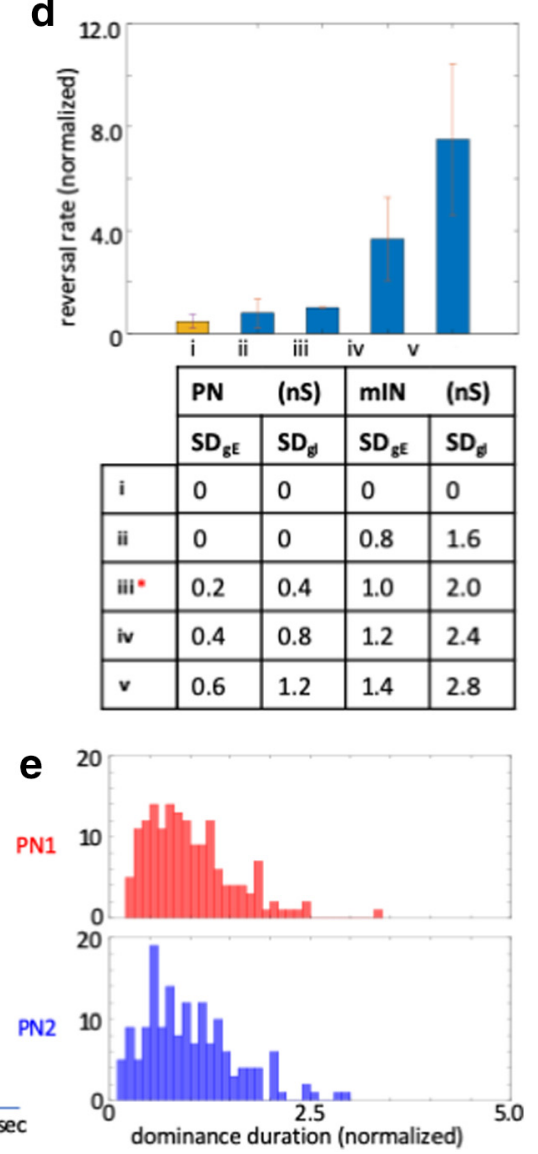

Figure 7. Effect of adding noise. Model excitatory and inhibitory synaptic noise was applied to the PNs and the INs through the dynamic clamp system. $\boldsymbol{a}, \boldsymbol{b}$, Baseline membrane potentials at $-60 \mathrm{mV}$ without $(\boldsymbol{a})$ and with $(\boldsymbol{b})$ the model noise. c, Effect of changing the noise level systematically to bistable activity. Increase of the noise resulted in increase of reversal rate (from top to bottom). Noise levels are indicated as SDs of $\mathrm{gE}$ and $\mathrm{gl}$ (excitatory and inhibitory conductance, respectively, in nS). *Data with the "standard" noise parameter set. $\boldsymbol{d}$, Pooled data of the effect of noise $(N=15)$. The reversal rates from the individual pair are normalized by the value at the standard noise parameters (iii) before pooling. Orange bar (i) indicates the data with no model noise. Error bars indicate \pm SEM. The noise parameter sets for $\mathrm{i}$ (no model noise), ii, iii (standard noise parameters), iv and $v$ are shown in the table below. The noise level is increased linearly from ii to $\mathrm{v}$. $\boldsymbol{e}$, Histogram of dominance durations for PN1 and PN2 from 10 min continuous recording (with the "standard" noise parameters).

primarily act to increase the average perceptual dominance duration of the stronger stimulus. Furthermore, the generalized Levelt's proposition II states that the change of stimulus intensity of the nondominant input is less effective. This means that, when the stimulus intensity changes from nondominant range to dominant range, the effect of the change to average dominant durations is weak in the nondominant range and strong in the dominant range. In Figure $8 d$, the change of the average dominance durations is plotted over the changing current for the example shown in Figure $8 b$. PN1 shows weak changes of the dominance durations on the left half of the plot where PN1 is weaker than PN2 (see Discussion). It shows, however, a steep increase on the right half of the plot where it is stronger than $\mathrm{PN} 2$, and vice versa for the other neuron. Hence, in general, the dominant neuron shows a steep increase of the dominance durations with current values deviating further away from $I_{50 \%}$. This trend can be seen in Figure $8 g$ with pooled data for the neurons whose currents were increased (red, $\left.F_{(6,24)}=4.371, p<0.01\right)$ and for their counterparts whose currents were kept constant (blue, $F_{(6,24)}=7.396, p<0.0001$ ). This is in line with the generalized Levelt's proposition II.

According to the generalized Levelt's proposition III: Increasing the difference in stimulus strength between the two competing stimuli will reduce the perceptual alternation rate. Figure $8 e$ plots the number of reversals for the example shown in Figure $8 b$. The pair showed a higher number of reversals for a current close to $I_{50 \%}$. Deviating further from $I_{50 \%}$ in either direction, the values decreased, in line with the generalized Levelt's proposition III. However, the pooled data (Fig. $8 h$ ) show that the response is not symmetric. Indeed, some pairs showed an increase of the reversal rate when a neuron is dominant (Fig. 9, bottom), in contrast to the example pair of Figure $8 b$ (and Fig. 9, top). Thus, the PN pairs did not always follow the generalized Levelt's proposition III. Because of the increase in the left half, repeated-measures ANOVA indicated a significant effect $\left(F_{(6,24)}=2.663, p<0.05\right)$.

To examine the generalized fourth proposition of Levelt, the currents injected into both neurons were varied. In total, 32 pairs were recorded with this paradigm. To pool the data, the change of the current is reported with reference to the current that would evoke $\sim 10 \mathrm{~Hz} \quad\left(I_{10 \mathrm{~Hz}}\right.$, see Materials and Methods).

Proposition IV states: Increasing stimulus strength of both competing stimuli will generally increase the perceptual alternation rate. In addition, the generalized proposition IV (Brascamp et al., 2015) noted that this effect may reverse at near-threshold stimulus strengths (i.e., the lower range of stimulation intensity). Figure $10 a$ shows an example of the effect of increasing the injected currents into both neurons. In Figure $10 b$, the number of reversals of this example are plotted over the injected current. Figure $10 c$ shows pooled data indicating increasing reversal rates $\left(F_{(6,30)}=4.051\right.$, $p<0.01)$. In addition, there is a small decrease of the reversal rate at the lower range of the stimulation. These results are in line with the generalized Levelt's proposition IV.

\section{Effect of short-term plasticity}

To explore the role of synaptic plasticity in neural competition and bistable activity, we implemented STD in the modeled excitatory and inhibitory synapses. Figure $11 a$ shows the depression of EPSPs evoked by a train of modeled presynaptic spikes (generated by SG, at $40 \mathrm{~Hz}$ ). Figure $11 b$ shows the results from a circuit with disynaptic inhibitory connections established between SG, 

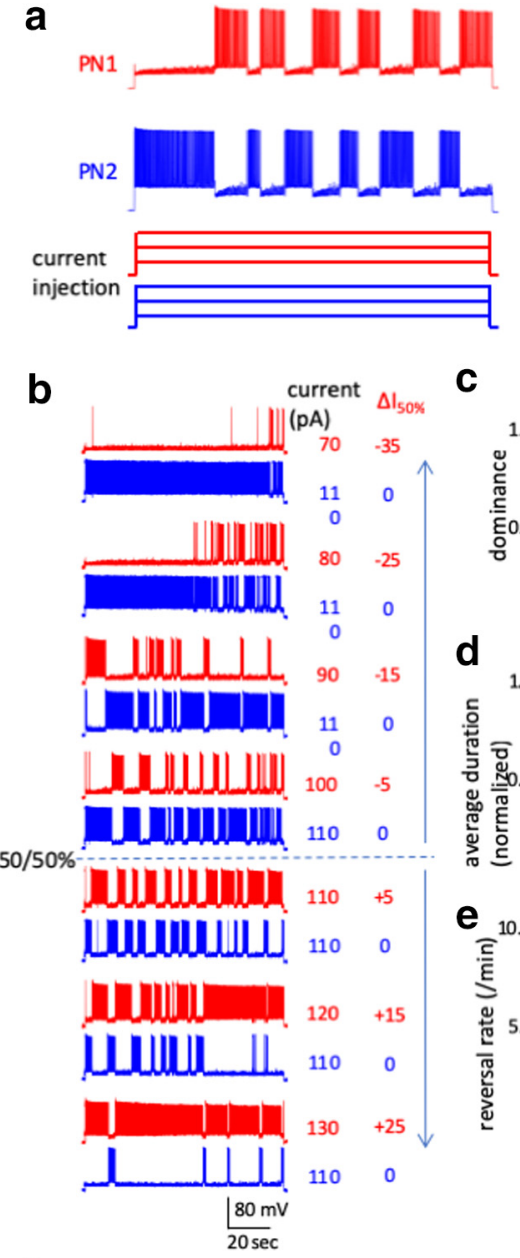

c

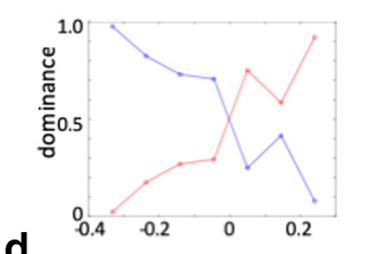

d
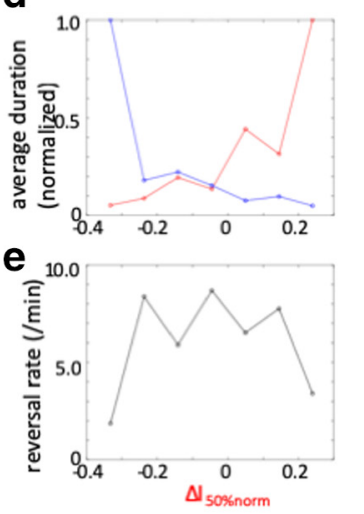

f

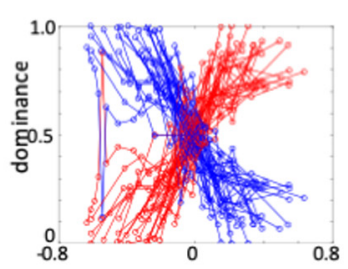

$\rightarrow$ neuron with changing current

$\rightarrow$ neuron without changing current

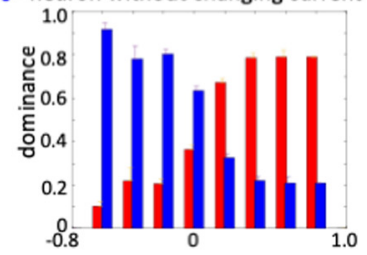

g

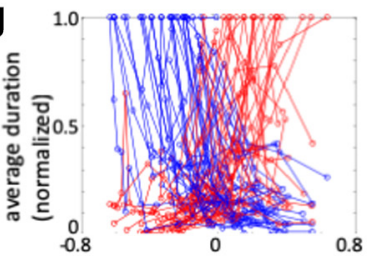

h

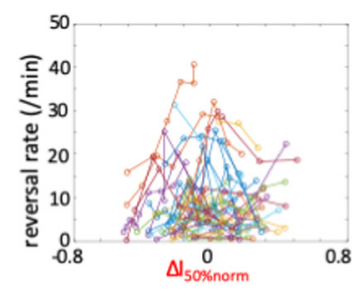

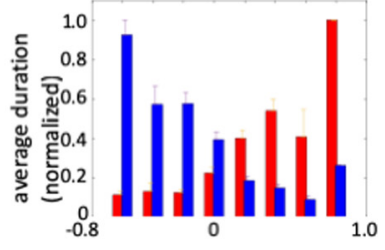

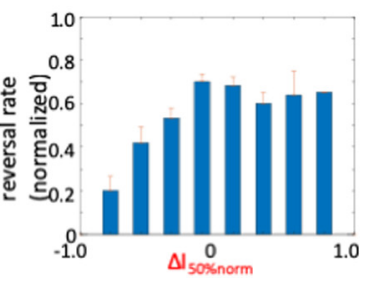

Figure 8. Schematics of experimental paradigm and the result. $\boldsymbol{a}$, Schematics of the paradigms equivalent to Levelt's experimental paradigms for bistable perception. The level of injected current to either one or both of the two mutually inhibiting PNs was systematically changed (analogous to the change of the contrasts in Levelt's experiments). $\boldsymbol{b}$, Example data of the experiment equivalent to the generalized Levelt's experimental paradigm for proposition I to III. The level of depolarization current in PN1 was increased (from top to bottom) while the current to PN2 was kept constant. $\mathbf{c}-\boldsymbol{e}$, Changes in dominance (c), dominance

mINs, and real-life PNs as illustrated in the schematic. The membrane potentials of the PNs were set to $-60 \mathrm{mV}$ to make IPSPs visible. Three different conditions were tested: without STD, with STD with strong synaptic inputs $\left(g_{s y n}=50 \mathrm{nS}\right)$, and with STD with weak synaptic inputs $\left(g_{s y n}=20 \mathrm{nS}\right)$, implemented in both EPSPs and IPSPs. A train of 10 spikes was generated at $20 \mathrm{~Hz}$ in the SG. EPSPs without STD successfully evoked action potentials in INs in response to all 10 EPSPs (left column). These action potentials, in turn, evoked long-lasting IPSPs in PNs (right column). When STD was implemented, the gradual decrease of EPSP size caused failures of evoking action potentials (marked by black asterisks) in INs. Compared with stronger EPSPs (middle traces), this effect was more pronounced with weaker EPSPs (bottom traces). The effect of implementing STD in PSPs as above on bistable activity is shown in Figure 11c. Bistable activity without STD is shown in the top traces. With STD with strong PSPs (middle traces), the pair of PNs showed bistability. However, because of the depression of EPSPs, action potentials in the dominant $\mathrm{PN}$ soon failed to evoke action potentials in the connected IN, and the reversal of dominance occurred quickly. As a result, the reversal rate became higher than the without STD condition. The ratio of reversal rate, with STD to without STD, was $8.6 \pm 4.2(N=9)$. In contrast, with STD with weak PSPs (bottom trances), action potentials in PNs failed to evoke action potentials in INs most of the time, and neither of the PNs was able to establish dominance in competition. Instead, both PNs showed continuous firing of action potentials without an apparent influence on the competing PNs $(N=7)$.

\section{Discussion}

We established a mutual inhibition between two real-life neurons mediated by dynamic clamp. This system enabled us to evoke bistable activity in PNs in visual cortex. We analyzed the dynamics of the bistability, a number of physiological properties, and the effects of manipulating the level of background noise and activation level. We compared the dynamics of this bistability with the known dynamics of human bistable perception. Although our experimental system represents the simplest neural unit of competition and human behavior represents the highly complex system, we found that the two systems show striking similarities in their dynamics.

The analyses of the physiological properties during bistable activity showed signs of adaptation of the dominant neurons. Moreover, the variations of interspike intervals and dominance durations were correlated, indicating a causal link between neural adaptation and the reversals in bistability. Neural adaptation has only been assumed as a key element for bistable perception theoretically (Matsuoka, 1984; Mueller, 1990; Wilson, 1999; Wilson et al., 2000; Laing and Chow, 2002; Lankheet, 2006; Noest et al., 2007; Shpiro et al., 2009) or it has been shown indirectly in the form of decreased contrast sensitivity (Alais et al., 2010). Our data directly show, in physiological terms, a

duration $(\boldsymbol{d})$, and reversal rate $(\boldsymbol{e})$ for this pair. Red represents PN1. Blue represents PN2. $\boldsymbol{f}-\boldsymbol{h}$, Pooled data $(N=46)$ plotted over the normalized injected current. The dominance durations are normalized for the maximum values of the individual neurons. Red represents responses of the neurons that received the changes of the injected current. Blue represents responses of the neurons whose injected current was kept constant. Left column represents the data of the individual pairs. Right column represents the normalized current was binned and the pooled data were averaged for the individual bins. Error bars indicate \pm SEM. 

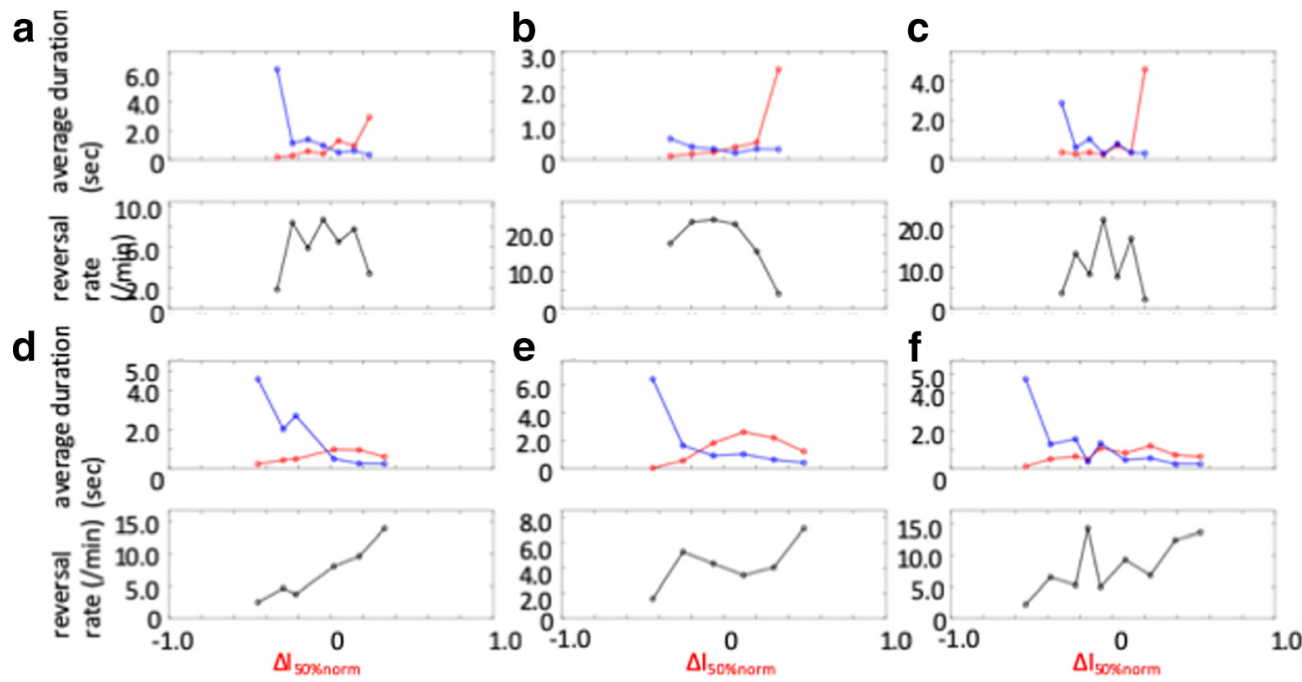

Figure 9. Examples of responses of average durations and reversal rate (shown in non-normalized absolute values) to the change of the depolarization current to one of the pair of PNs (red) while the current to the other neurons was kept constant (blue). $\boldsymbol{a}$-c, The example pairs where the reversal rates decreased when the current either increased or decreased from the control value $\left(I_{50 \%}\right)$. $\boldsymbol{d}-\boldsymbol{f}$, The examples where the reversal rates increased when the current increased from the control value $\left(I_{50 \%}\right)$. In the latter case, the increase of the average.

a

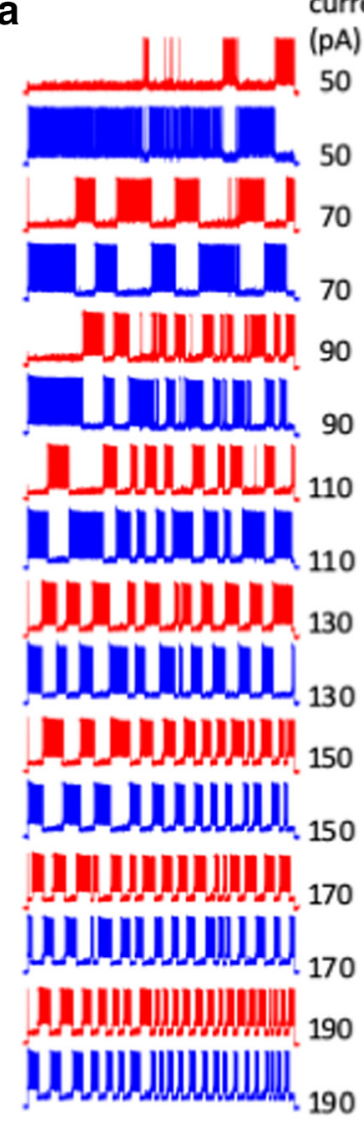

current $\quad b$

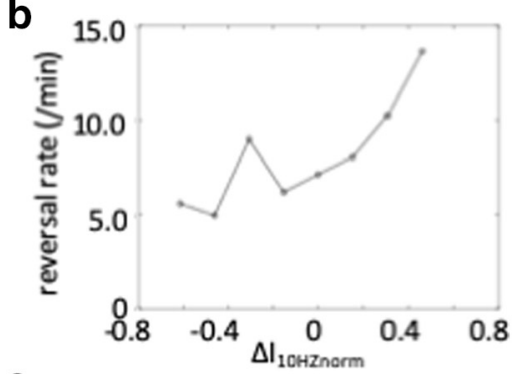

C
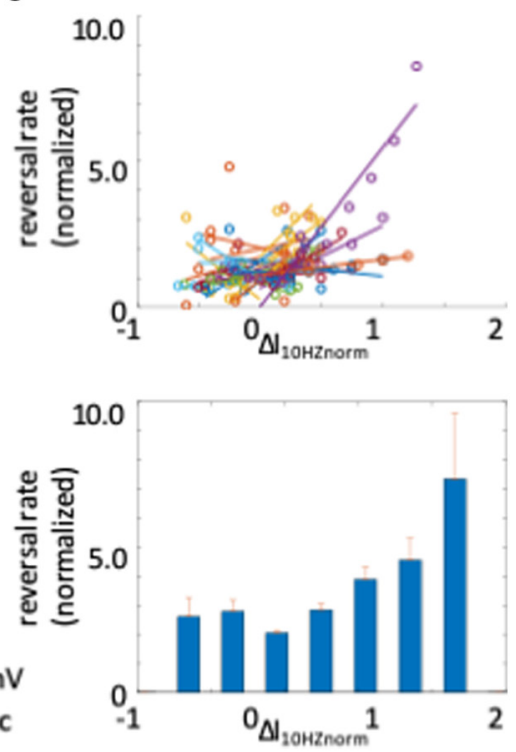

Figure 10. Results of experimental paradigm equivalent to the generalized Levelt's paradigm for proposition IV. $\boldsymbol{a}$, The effect of increasing the depolarization currents simultaneously in both PNs (from top to bottom). $\boldsymbol{b}$, The changes of the reversal rate for this pair. $c$, Pooled data of reversal rate $(N=32)$ plotted over the normalized injected currents.

progression of adaptation during bistable activity and its link to the dominance durations.

We investigated the effect of neural noise on the dynamics of bistability. The apparent stochasticity in the sequence of reversals

and the skewed distribution of dominance durations (Levelt, 1967) in bistable perception led to studies on the role of noise (Brascamp et al., 2006; Kim et al., 2006; Moreno-Bote et al., 2007; Huguet et al., 2014; Pisarchik et al., 2014; Baker and Richard, 2019). To investigate the effect of noise in our experimental model, we incorporated a neurocomputational model of synaptic noise into the dynamic clamp. In this way, we were able to insert noise into neurons and systematically changed the level of noise. We found that an increase of noise caused an increase of reversal rate. It is known that the synaptic noise in brain slice preparations is much less than the noise in intact brains (Destexhe et al., 2001) or in human brain tissue (Molnár et al., 2008). Hence, we added noise levels equivalent to the noise level in the intact brain (Destexhe et al., 2001). We found that the histogram of dominance durations was rightskewed as is typically found in bistable perception.

We showed that, when one of the two neurons is dominant, its adaptation progresses and hence the interspike interval increases over time. This allows the suppressed neuron to recover from its own adaption and to depolarize more during the ever-increasing interspike intervals of the dominant neuron, consequently showing a slowly ramping depolarization. When the membrane potential comes close to the firing threshold, the noise facilitates the membrane potential to go above the threshold, generating action potentials. As a consequence, the dominant neuron now receives IPSPs and a reversal occurs. Hence, our data elucidate the dynamic interplay between adaptation, noise, and mutual inhibition in determining the dynamics of bistable activity.

Our experimental model allowed us to separately manipulate the levels of activation of the competing neurons. Hence, it enabled us to compare the effects of changing activation levels in neurons to the effects of changes in stimulus strength on bistable perception, as originally 
described in Levelt's four propositions (Levelt, 1965). Levelt's propositions I, II, and III make predictions about the changes of dominance, the dominance durations, and the reversal rate, respectively, in response to changes of the stimulation strength in one of the two inputs. Levelt's proposition IV concerns the change in the reversal rate while the stimulus strengths of both inputs are changed concurrently. The original propositions were modified later (Brascamp et al., 2006, 2015; Moreno-Bote et al., 2010) to cover the whole range of the stimulus strength (dominant and nondominant ranges). By running paradigms equivalent to these experiments, we found that both systems show striking similarities in their dynamics.

It is quite intriguing that, although the overall effect of increasing the injected current was the increase of the reversal rate in the paradigm for the generalized Levelt's proposition IV, we observed a small decrease of it in the lower range of the injected currents. A small deviation of the response from the original proposition by Levelt has been reported by several papers (Shpiro et al., 2007; Curtu et al., 2008; Seely and Chow, 2011; see Brascamp et al., 2015). In our experiment, when the injection current was lowered, generation of action potentials by the dominant neuron became sporadic. As a result, the spike interval became longer, giving room to the suppressed neuron to recover from the inhibition and reach the threshold of action potentials. On the one hand, in the higher range of injection currents, the reversal occurred because spike intervals of the dominant neuron gradually increased because of adaptation. On the other hand, in the lower range of injection currents, the reversal occurred because of the lower frequency of evoked action potentials. The latter may be potentially a mechanism underlying the small decrease in the lower range of stimulus reported in bistable perception.

One exception where our data did not necessarily match the known dynamics of bistable perception was the mixed results for the Levelt III paradigm. In this paradigm, some neuron pairs showed a decrease of reversal rates when the depolarization current either increased or decreased from the control value, which is in line with the generalized Levelt's proposition III. However, other pairs showed no significant change or an increase of reversal rate when the current was higher than the control. The mixed results suggest involvement of multiple factors. The reversal rate is determined by the balance between increased dominance durations of the stronger neuron and decreased dominance durations of the weaker neuron.
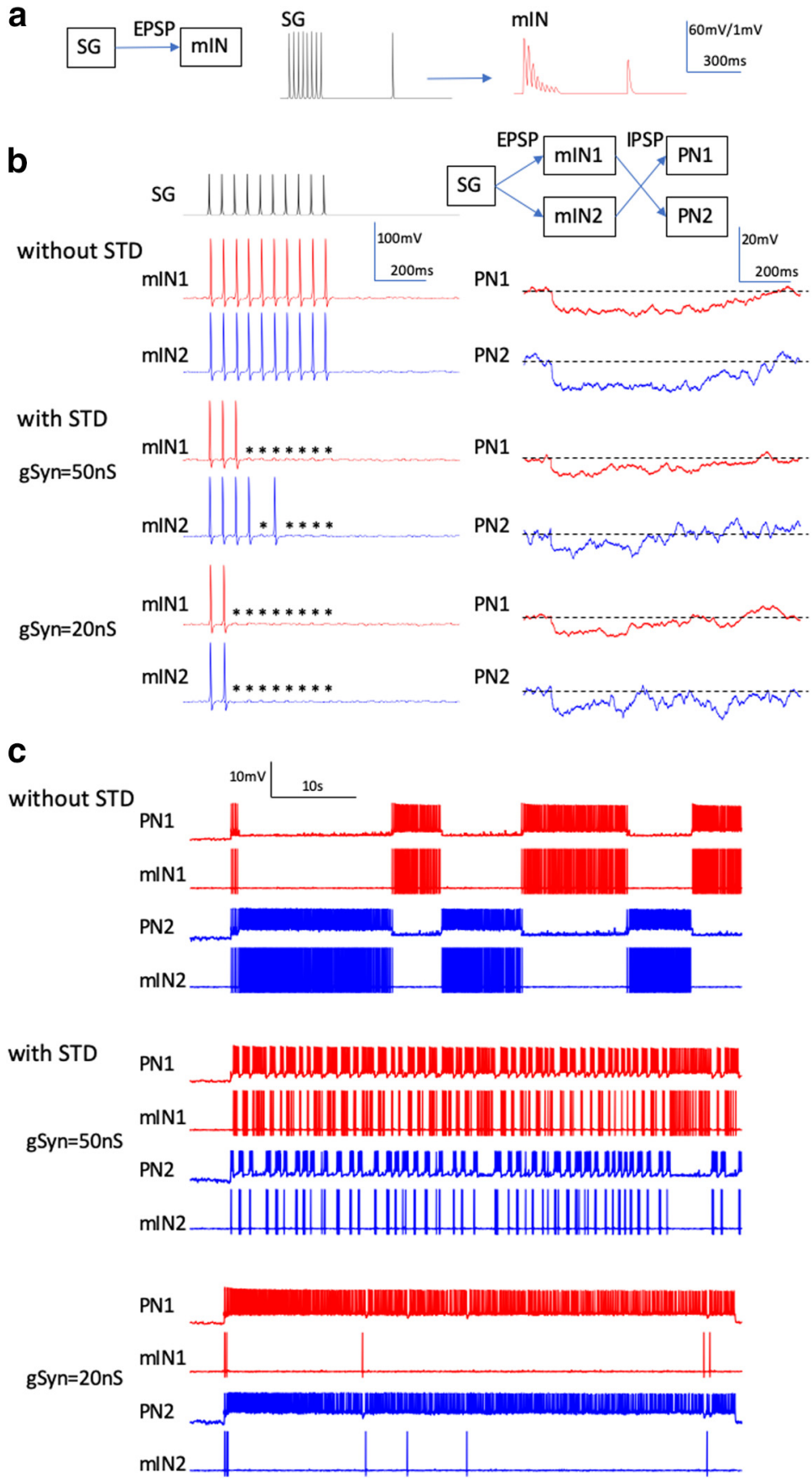

Figure 11. Effect of STD on bistable activity. $\boldsymbol{a}$, Depression of EPSPs evoked by a train of modeled presynaptic spikes (by $\mathrm{SG}$, at $40 \mathrm{~Hz}$ ). $g_{\text {syn }}$ was set to $5 \mathrm{nS}$ to avoid generation of action potentials for illustration purposes. $\boldsymbol{b}$, A disynaptic inhibitory circuit was established between SG, mINs, and real-life PNs as illustrated in the schematic (top). The membrane potentials of PNs were set to $-60 \mathrm{mV}$ to make IPSPs visible. Three different conditions were tested, including no STD (top), and STD with strong $\left(g_{\text {syn }}=50 \mathrm{nS}\right.$, middle) and weak $\left(g_{\text {syn }}=20 \mathrm{nS}\right.$, bottom) synapses in both the inhibitory and excitatory connections. A train of 10 spikes was generated in the SG at $20 \mathrm{~Hz}$. Without STD, all 10 EPSPs successfully evoked action potentials in INs (left column). These action potentials, in turn, evoked long-lasting IPSPs in PNs (right column). With STD (strong and weak synaptic strength, middle and bottom traces, respectively), the gradual decrease of EPSP size caused failures of evoking action potentials in INs (asterisks), resulting in weaker IPSPs in the target PNs. c, An example of the effect of STD on bistable activity. Without STD (top), with STD with strong synapses (middle) and with weak synapses (bottom). With STD with strong synapses, action potentials in the dominant PN soon failed to evoke action potentials in the connected IN because of the depression of EPSPs and reversal of dominance occurred quickly. With STD with weak synapses, EPSPs evoked by action potentials in PNs failed to evoke action potentials in INs most of the time, neither of the PNs was able to establish dominance, and both PNs showed continuous firing of action potentials independently. 
If the former is more significant, the reversal rate will decrease; and if the latter is more significant, it will increase. The increase of the firing rate in the stronger neuron may cause a stronger dominance of the neuron on one hand, and a stronger adaptation of the neuron on the other hand. The latter may prevent the increase of the dominant durations because of the faster decay of the firing rate. Hence, depending on the adaptation properties and the spiking properties of the neurons, the strong activation of the stronger neuron may have caused a decrease of the reversal rate in some cases and an increase in other cases. At systems level, the competition is between populations of neurons rather than single neurons as tested here. Hence, differences in adaptation and spiking properties among the involved neurons may collectively have different impacts on the dynamics of bistability. Furthermore, in the human brain, the input signals go through multiple steps of normalization before reaching the mutual inhibition processes. It may be possible that the activation level of neurons in the human visual system is kept within the range where the fast adaptation occurs in a lesser amount. If this is the case, the strong stimulation would cause the decrease of the reversal rate as reported in the generalized Levelt III. Therefore, this result may represent an example where emergent properties of bistable perception at the behavioral level differ from the dynamics found in the minimal neural competition unit we investigated.

Regarding the dominance durations, there are short periods when the neuron that has been suppressed fires only one or two action potentials and then becomes suppressed again. Such short events are not considered as a reversal in our analyses, and the dominance durations are determined by neglecting these events (see Fig. 3). Furthermore, there are periods where short events occurred alternatingly between the two neurons with intermingled action potentials from both neurons. In these periods, neither of the two neurons is considered to be dominant. These observations may be linked to known observations in bistable perception. It has been reported that human subjects experience short reversal events detected in reflexes (optokinetic nystagmus and pupil dilations), but they are too short to be reported by the subjects (Naber et al., 2011). Furthermore, the intermingled firing of action potentials by the two neurons may be related to the period in bistable perception where the perception of the subject is either uncertain or a mixture of the two possible percepts ("composite" or "mixed" perception). The short and the mixture events are potentially important because they may elucidate the neural mechanisms underlying the stochastic properties of bistability and decision-making processes. Hence, this intriguing property of bistable neural activity during the transition of the dominances should be investigated further in the future.

Finally, it is important to consider the diversity of neural properties in neocortex. First, in terms of the location where neural competition relevant to bistable perception takes place, it is unknown which layer and which area of visual cortex are involved. It is also possible that the final decision is the result of integrating neural competition at multiple levels. In addition to the issue of location, neurons display a range of firing patterns even within the same area and the same layer. At the onset of activation, for example, an early bursting-type PN may have an advantage over a regular-spiking-type $\mathrm{PN}$ to win the onset dominance. Different adaptation properties influence how the reversal of dominance occurs as well. The differences of these properties are present not only in PNs but also in INs (e.g., nonadapting fast spiking neurons or adapting-type regular spiking neurons). Furthermore, cell-type-specific short-term plasticity has been shown. Therefore, it is important to diversify the research on neural competition reported here by applying our approach to neurons from different layers with different firing patterns, adaptation properties, and synaptic properties. How details of the dynamics of bistability change depending on these neurophysiological differences remains to be elucidated by future research.

In conclusion, our experimental model provides a platform for investigating the dynamics of a theoretically derived neural circuit in real-life neurons. Our data showed that even the simplest neural competition circuit already reproduces many aspects of dynamics of bistable perception in human perception. Our study using the novel approach reported here provides a platform to investigate further how elementary neural competition units are integrated to execute system-level bistable dynamics.

\section{References}

Alais D, Cass J, O'Shea RP, Blake R (2010) Visual sensitivity underlying changes in visual consciousness. Curr Biol 20:1362-1367.

Baker DH, Richard B (2019) Dynamic properties of internal noise probed by modulating binocular rivalry. PLoS Comput Biol 15:e1007071.

Beck DM, Kastner S (2005) Stimulus context modulates competition in human extrastriate cortex. Nat Neurosci 8:1110-1116.

Berger TK, Perin R, Silberberg G, Markram H (2009) Frequency-dependent disynaptic inhibition in the pyramidal network: a ubiquitous pathway in the developing rat neocortex. J Physiol 587:5411-5425.

Bonds AB (1989) Role of inhibition in the specification of orientation selectivity of cells in the cat striate cortex. Vis Neurosci 2:41-55.

Brascamp JW, van Ee R, Noest AJ, Jacobs RH, van den Berg AV (2006) The time course of binocular rivalry reveals a fundamental role of noise. J Vis 6:1244-1256.

Brascamp JW, Klink PC, Levelt WJ (2015) The "laws" of binocular rivalry: 50 years of Levelt's propositions. Vision Res 109:20-37.

Curtu R, Shpiro A, Rubin N, Rinzel J (2008) Mechanisms for frequency control in neuronal competition models. SIAM J Appl Dyn Syst 7:609-649.

Delgado JY, Gómez-González JF, Desai NS (2010) Pyramidal neuron conductance state gates spike-timing-dependent plasticity. J Neurosci 30:15713-15725.

Destexhe A, Rudolph M, Fellous JM, Sejnowski TJ (2001) Fluctuating synaptic conductances recreate in vivo-like activity in neocortical neurons. Neuroscience 107:13-24.

Dong H, Shao Z, Nerbonne JM, Burkhalter A (2004) Differential depression of inhibitory synaptic responses in feedforward and feedback circuits between different areas of mouse visual cortex. J Comp Neurol 475:361373.

Hayden BY, Moreno-Bote R (2018) A neuronal theory of sequential economic choice. Brain Neurosci Adv 2:2398212818766675.

Heuer H (1987) Visual discrimination and response programming. Psychol Res 49:91-98.

Hodgkin AL, Huxley AF (1952) A quantitative description of membrane current and its application to conduction and excitation in nerve. J Physiol 117:500-544.

Huguet G, Rinzel J, Hupé JM (2014) Noise and adaptation in multistable perception: noise drives when to switch, adaptation determines percept choice. J Vis 14:19.

Kapfer C, Glickfeld LL, Atallah BV, Scanziani M (2007) Supralinear increase of recurrent inhibition during sparse activity in the somatosensory cortex. Nat Neurosci 10:743-753.

Kemenes I, Marra V, Crossley M, Samu D, Staras K, Kemenes G, Nowotny T (2011) Dynamic clamp with StdpC software. Nat Protoc 6:405-417.

Kim YJ, Grabowecky M, Suzuki S (2006) Stochastic resonance in binocular rivalry. Vision Res 46:392-406.

Kogo N, van Ee R (2015) Neural mechanisms of figure-ground organization: border-ownership, competition and perceptual switching. In: Oxford handbook of perceptual organization, pp 342-362. Oxford: Oxford UP.

Laing CR, Chow CC (2002) A spiking neuron model for binocular rivalry. J Comput Neurosci 12:39-53.

Lankheet MJM (2006) Unraveling adaptation and mutual inhibition in perceptual rivalry. J Vis 6:304-310. 
Lee DK, Itti L, Koch C, Braun J (1999) Attention activates winner-take-all competition among visual filters. Nat Neurosci 2:375-381.

Leopold DA, Logothetis NK (1999) Multistable phenomena: changing views in perception. Trends Cogn Sci 3:254-264.

Levelt WJ (1965) On binocular rivalry. Soesterberg, The Netherlands: Institute for Perception RVO-TNO.

Levelt WJ (1967) Note on the distribution of dominance times in binocular rivalry. Br J Psychol 58:143-145.

Lien CC, Jonas P (2003) Kv3 potassium conductance is necessary and kinetically optimized for high-frequency action potential generation in hippocampal interneurons. J Neurosci 23:2058-2068.

Machens CK, Romo R, Brody CD (2005) Flexible control of mutual inhibition: a neural model of two-interval discrimination. Science 307:11211124.

Mark S, Romani S, Jezek K, Tsodyks M (2017) Theta-paced flickering between place-cell maps in the hippocampus: a model based on shortterm synaptic plasticity. Hippocampus 27:959-970.

Markram H, Toledo-Rodriguez M, Wang Y, Gupta A, Silberberg G, Wu C (2004) Interneurons of the neocortical inhibitory system. Nat Rev Neurosci 5:793-807.

Masquelier T, Guyonneau R, Thorpe SJ (2009) Competitive STDP-based spike pattern learning. Neural Comput 21:1259-1276.

Matsuoka K (1984) The dynamic model of binocular rivalry. Biol Cybern 49:201-208

Mikami A, Newsome WT, Wurtz RH (1986) Motion selectivity in macaque visual cortex: I. Mechanisms of direction and speed selectivity in extrastriate area MT. J Neurophysiol 55:1308-1327.

Miles R (1990) Synaptic excitation of inhibitory cells by single CA3 hippocampal pyramidal cells of the guinea-pig in vitro. J Physiol 428:61-77.

Molnár G, Oláh S, Komlósi G, Füle M, Szabadics J, Varga C, Barzó P, Tamás $\mathrm{G}$ (2008) Complex events initiated by individual spikes in the human cerebral cortex. PLoS Biol 6:e222.

Moreno-Bote R, Rinzel J, Rubin N (2007) Noise-induced alternations in an attractor network model of perceptual bistability. J Neurophysiol 98:1125-1139.

Moreno-Bote R, Shpiro A, Rinzel J, Rubin N (2010) Alternation rate in perceptual bistability is maximal at and symmetric around equi-dominance. J Vis 10:1.

Mueller TJ (1990) A physiological model of binocular rivalry. Vis Neurosci 4:63-73.

Naber M, Frässle S, Einhäuser W (2011) Perceptual rivalry: reflexes reveal the gradual nature of visual awareness. PLoS One 6:e20910.

Noest AJ, van Ee R, Nijs MM, van Wezel RJ (2007) Percept-choice sequences driven by interrupted ambiguous stimuli: a low-level neural model. J Vis 7:10 1-14.

Nowotny T, Szucs A, Pinto RD, Selverston AI (2006) StdpC: a modern dynamic clamp. J Neurosci Methods 158:287-299.

Pastukhov A, Braun J (2011) Cumulative history quantifies the role of neural adaptation in multistable perception. J Vis 11:12.
Pelkey KA, Chittajallu R, Craig MT, Tricoire L, Wester JC, McBain CJ (2017) Hippocampal GABAergic inhibitory interneurons. Physiol Rev 97:16191747.

Pisarchik AN, Jaimes-Reátegui R, Magallón-García CD, Castillo-Morales CO (2014) Critical slowing down and noise-induced intermittency in bistable perception: bifurcation analysis. Biol Cybern 108:397-404.

Pospischil M, Toledo-Rodriguez M, Monier C, Piwkowska Z, Bal T, Frégnac Y, Markram H, Destexhe A (2008) Minimal Hodgkin-Huxley type models for different classes of cortical and thalamic neurons. Biol Cybern 99:427-441.

Ren M, Yoshimura Y, Takada N, Horibe S, Komatsu Y (2007) Specialized inhibitory synaptic actions between nearby neocortical pyramidal neurons. Science 316:758-761.

Said CP, Heeger DJ (2013) A model of binocular rivalry and cross-orientation suppression. PLoS Comput Biol 9:e1002991.

Seely J, Chow CC (2011) Role of mutual inhibition in binocular rivalry. J Neurophysiol 106:2136-2150.

Shpiro A, Curtu R, Rinzel J, Rubin N (2007) Dynamical characteristics common to neuronal competition models. J Neurophysiol 97:462-473.

Shpiro A, Moreno-Bote R, Rubin N, Rinzel J (2009) Balance between noise and adaptation in competition models of perceptual bistability. J Comput Neurosci 27:37-54

Silberberg G, Markram H (2007) Disynaptic inhibition between neocortical pyramidal cells mediated by Martinotti cells. Neuron 53:735-746.

Silberberg G, Grillner S, Lebeau F, Maex R, Markram H (2005) Synaptic pathways in neural microcircuits. Trends Neurosci 28:541-551.

Sillito AM (1975) The contribution of inhibitory mechanisms to the receptive field properties of neurones in the striate cortex of the cat. J Physiol 250:305-329.

Snowden RJ, Treue S, Erickson RG, Andersen RA (1991) The response of area MT and V1 neurons to transparent motion. J Neurosci 11:27682785.

Tremblay R, Lee S, Rudy B (2016) GABAergic interneurons in the neocortex: from cellular properties to circuits. Neuron 91:260-292.

Uhlenbeck GE, Ornstein LS (1930) On the theory of the Brownian motion. Phys Rev 36:823-841.

Usher M, McClelland JL (2001) The time course of perceptual choice: the leaky, competing accumulator model. Psychol Rev 108:550-592.

Wang CT, Lee CT, Wang XJ, Lo CC (2013) Top-down modulation on perceptual decision with balanced inhibition through feedforward and feedback inhibitory neurons. PLoS One 8:e62379.

Wang Q, Burkhalter A (2007) Area map of mouse visual cortex. J Comp Neurol 502:339-357.

Wilson HR (1999) Spikes, decisions, and actions: the dynamical foundations of neuroscience. Oxford: Oxford UP

Wilson HR, Krupa B, Wilkinson F (2000) Dynamics of perceptual oscillations in form vision. Nat Neurosci 3:170-176.

Zhou H, Friedman HS, von der Heydt R (2000) Coding of border ownership in monkey visual cortex. J Neurosci 20:6594-6611. 